nucl-th/0512063; TUM-T39-05-18; FAU-TP3-05/9

Revised version 3rd August 2010.

\title{
Nucleon Polarizabilities from Deuteron Compton Scattering within a Green's-Function Hybrid Approach
}

\author{
Robert P. Hildebrandt ${ }^{a}$, Harald W. Grießhammer ${ }^{a, b, c, 1}$ \\ and Thomas R. Hemmert ${ }^{a}$ \\ a Institut für Theoretische Physik (T39), Physik-Department, \\ Technische Universität München, D-85747 Garching, Germany \\ ${ }^{b}$ Institut für Theoretische Physik III, Naturwissenschaftliche Fakultät I, \\ Universität Erlangen-Nürnberg, D-91058 Erlangen, Germany \\ ${ }^{c}$ Center for Nuclear Studies, Department of Physics, \\ The George Washington University, Washington DC 20052, USA
}

\begin{abstract}
We examine elastic Compton scattering from the deuteron for photon energies ranging from zero to $100 \mathrm{MeV}$, using state-of-the-art deuteron wave functions and $N N$-potentials. Nucleon-nucleon rescattering between emission and absorption of the two photons is treated by Green's functions in order to ensure gauge invariance and the correct Thomson limit. With this Green's-function hybrid approach, we fulfill the low-energy theorem of deuteron Compton scattering and there is no significant dependence on the deuteron wave function used. Concerning the nucleon structure, we use Chiral Effective Field Theory with explicit $\Delta(1232)$ degrees of freedom within the Small Scale Expansion up to leading-one-loop order. Agreement with available data is good at all energies. Our 2-parameter fit to all elastic $\gamma d$ data leads to values for the static isoscalar dipole polarizabilities which are in excellent agreement with the isoscalar Baldin sum rule. Taking this value as additional input, we find $\alpha_{E}^{s}=$ $(11.3 \pm 0.7$ (stat) \pm 0.6 (Baldin) \pm 1 (theory) $) \cdot 10^{-4} \mathrm{fm}^{3}$ and $\beta_{M}^{s}=(3.2 \mp 0.7$ (stat) \pm 0.6 (Baldin) \pm 1 (theory) $) \cdot 10^{-4} \mathrm{fm}^{3}$ and conclude by comparison to the proton numbers that neutron and proton polarizabilities are the same within rather small errors.
\end{abstract}

Suggested PACS numbers: 13.40.-f, 13.60.Fz, 25.20.-x, 21.45.+v

Suggested Keywords: Effective Field Theory, Compton Scattering, Deuteron, Delta Resonance, Nucleon Polarizabilities.

\footnotetext{
${ }^{1}$ Corresponding author; email: hgrie@gwu.edu; permanent address: c
} 


\section{Introduction}

Analyzing protons and neutrons with electromagnetic probes has a long history in the field of nucleon-structure studies. In Compton scattering off a single nucleon, the electromagnetic field of the scattered photon attempts to deform the nucleon. The global resistance of the nucleon's internal degrees of freedom against this deformation is measured in terms of the electromagnetic polarizabilities, which makes them an excellent tool to study the structure of the nucleon. In principle, each polarizability is a function of the frequency of the electromagnetic wave. Therefore, energy-dependent polarizabilities have been introduced in Refs. [1,2]. In this work, we determine the static values of the nucleon polarizabilities from experiment, i.e. the values in the limit of vanishing photon energy, which we therefore denote as the polarizabilities for simplicity. The two most prominent nucleon polarizabilities are the static electric and magnetic dipole polarizabilities $\alpha_{E}$ and $\beta_{M}$. For the proton, rather precise experimental values for these two quantities exist, e.g. those given in a recent review [3], which were obtained as the weighted average over several experiments,

$$
\alpha_{E}^{p}=(12.0 \pm 0.6) \cdot 10^{-4} \mathrm{fm}^{3}, \quad \beta_{M}^{p}=(1.9 \mp 0.6) \cdot 10^{-4} \mathrm{fm}^{3} .
$$

Our recent fit to the proton Compton data yielded [2]

$$
\begin{aligned}
& \alpha_{E}^{p}=(11.04 \pm 1.36(\text { stat }) \pm 0.4(\text { Baldin })) \cdot 10^{-4} \mathrm{fm}^{3}, \\
& \beta_{M}^{p}=(2.76 \mp 1.36(\text { stat }) \pm 0.4(\text { Baldin })) \cdot 10^{-4} \mathrm{fm}^{3},
\end{aligned}
$$

in agreement with Eq. (1.1), and with the central value of the Baldin sum rule $\alpha_{E}^{p}+\beta_{M}^{p}=$ $(13.8 \pm 0.4) \cdot 10^{-4} \mathrm{fm}^{3}$ [4]. The framework that has been chosen for this extraction is third-order $\left(\mathcal{O}\left(\epsilon^{3}\right)\right)$ Small Scale Expansion (SSE), which is one possible way to include the $\Delta(1232)$ resonance explicitly in Chiral Effective Field Theory, cf. Ref. [5, 6]. It is this very framework on which the one-nucleon sector of our present work is built.

On the other hand, neutron polarizabilities are much harder to access experimentally, as there is no stable single-neutron target for Compton scattering. Therefore, one has to rely on other methods in order to investigate these quantities. One approach is quasi-free Compton scattering off the neutron bound in the deuteron, or the scattering of neutrons from a lead target. The weighted average over several experiments investigating these two processes gives the result [3]

$$
\alpha_{E}^{n}=(12.5 \pm 1.7) \cdot 10^{-4} \mathrm{fm}^{3}, \quad \beta_{M}^{n}=(2.7 \mp 1.8) \cdot 10^{-4} \mathrm{fm}^{3} .
$$

The numbers given in Eq. (1.3) do not include information from elastic low-energy Compton scattering from light nuclei, as these processes so far led to values inconsistent with Eq. (1.3). However, several such experiments have already been performed [7 9] and further proposals exist, e.g. from the proton, deuteron or ${ }^{3} \mathrm{He}$ at TUNL/HI $\gamma \mathrm{S}$ [10 15] and on deuteron targets at MAXlab [16,17] as well as at the S-DALINAC [18], and for the proton at MAMI [19]. They promise an extensive study of elastic deuteron Compton scattering below the pion-production threshold. From a theorist's point of view, extracting the neutron polarizabilities requires an accurate description both of the structure of the nucleon 
and of the dynamics of the low-energy degrees of freedom within the deuteron, as one has to account for the nucleon polarizabilities as well as for meson-exchange currents. We remind the reader that the deuteron as the proton-neutron bound state is an isoscalar object, so that only $\alpha_{E}^{s} \equiv \frac{1}{2}\left(\alpha_{E}^{p}+\alpha_{E}^{n}\right), \beta_{M}^{s} \equiv \frac{1}{2}\left(\beta_{M}^{p}+\beta_{M}^{n}\right)$ is measured. The isovector polarizabilities are defined as $\alpha_{E}^{v} \equiv \frac{1}{2}\left(\alpha_{E}^{p}-\alpha_{E}^{n}\right), \beta_{M}^{v} \equiv \frac{1}{2}\left(\beta_{M}^{p}-\beta_{M}^{n}\right)$. A first attempt to fit the isoscalar polarizabilities to the elastic deuteron Compton-scattering data from Illinois [7] and SAL [9] was made in [20]. The extracted neutron polarizabilities $\alpha_{E}^{n}=(9.0 \pm 3.0) \cdot 10^{-4} \mathrm{fm}^{3}$, $\beta_{M}^{n}=(11.0 \pm 3.0) \cdot 10^{-4} \mathrm{fm}^{3}$ indicated the possibility of a rather large isovector part, especially in the magnetic dipole polarizability, in contrast to the quasi-elastic result from [21] contained in Eq. (1.3). The enhancement of $\beta_{M}^{n}$ in [20] is in our opinion due to an insufficient description of the SAL-data around $94 \mathrm{MeV}$, in particular in the backward direction, which have been a puzzle for several years. It was finally resolved by the authors of [22] in an Effective-Field-Theory calculation by observing that dynamical effects from explicit $\Delta(1232)$ resonance degrees of freedom are large at these energies. The calculation presented in [22] agrees reasonably with data but only works above some lower energy limit of the order 50-60 MeV. The "best" (Baldin-constrained) fit results for the isoscalar polarizabilities given in [22] are in excellent agreement with Eqs. (1.1) and (1.3):

$$
\begin{aligned}
& \alpha_{E}^{s}=(12.6 \pm 0.8(\text { stat }) \pm 0.7(\mathrm{wf}) \pm 0.6(\text { Baldin })) \cdot 10^{-4} \mathrm{fm}^{3}, \\
& \beta_{M}^{s}=(1.9 \mp 0.8(\text { stat }) \mp 0.7(\mathrm{wf}) \pm 0.6(\text { Baldin })) \cdot 10^{-4} \mathrm{fm}^{3},
\end{aligned}
$$

with 'wf' denoting the uncertainty arising from the residual wave-function dependence.

On the theory side, Heavy Baryon Chiral Perturbation Theory (HB $\chi \mathrm{PT}$ ) predicts that the proton and neutron polarizabilities are equal at leading-one-loop order [23]. Comparing Eq. (1.1) to (1.3) or (1.4), respectively, suggests that $\alpha_{E}$ and $\beta_{M}$ have indeed only small isovectorial components. A thorough discussion and review of the chiral aspects of Compton scattering can for example be found in Ref. 24] and references therein.

Let us first sketch where our approach agrees or differs from other calculations, and which problems we hope to solve by such modifications. In the present work, we investigate elastic deuteron Compton scattering including photon couplings to the one-pion exchange. Diagrams which are characterized by the propagation of the nucleons between the two photon interactions are calculated using Green's-function methods in analogy to Ref. [25,26]. The single-nucleon structure is included within Chiral Effective Field Theory $(\chi \mathrm{EFT})$ with explicit $\Delta$ degrees of freedom up to leading-one-loop order like in Ref. [22], where isovectorial components are zero. The $\Delta(1232)$ is treated according to the power-counting rules of the Small Scale Expansion [5, 6]. We aim for a consistent calculation at photon energies $\omega$ below $100 \mathrm{MeV}$, i.e. we

1) give an improved description of the high-energy $(\omega \sim 95 \mathrm{MeV})$ data from [9] with respect to the calculations presented in [20,25, 26].

2) present an alternative approach that does not suffer from the shortcomings of the EFT calculations of Refs. [22, 27, 29], which are not applicable below some lower energy limit. Stated differently, we demand that our calculation reaches the correct static limit, i.e. the Thomson limit for deuteron Compton scattering. 
3) contribute to the ongoing discussion of the neutron polarizabilities via fits of the isoscalar polarizabilities $\alpha_{E}^{s}$ and $\beta_{M}^{s}$ to all existing elastic deuteron Compton data.

Effective Field Theory has already been used for the latter purpose in [22] and [28, 29]. The authors of [22, 28, 29], as well as those of Ref. [27], followed Weinberg's proposal [30, 31] to calculate the irreducible kernel for the $\gamma N N \rightarrow \gamma N N$ process in Effective Field Theory and then folded this with external deuteron wave functions, derived from high-precision NN-potentials such as Nijm93 [32, CD-Bonn [33] or AV18 [34. This "hybrid" approach has proven quite successful in describing scattering reactions like $\pi d$ [35], $e^{-} d$ [36] and other processes, see e.g. also [37. However, the $\mathcal{O}\left(p^{4}\right)-\mathrm{HB} \chi \mathrm{PT}$ calculation of Ref. [28, 29] in the Weinberg hybrid approach gives an insufficient description of the SAL-data [9] measured in the backward direction. Therefore, the authors of [28,29] excluded these (two) data points in some of their fits. Those of Ref. 22] had to restrict themselves to the published data above $60 \mathrm{MeV}$. It is one of the central results of the approach presented in this work that we do not need any such constraints, in particular in view of forthcoming data even beyond $\omega=100 \mathrm{MeV}$ [16, 17].

In this work, we present an extended hybrid approach, called the Green's function hybrid approach. It includes the full two-nucleon Green's function in all diagrams with an $N N$ intermediate state, which was only treated perturbatively in Ref. [22], according to the power-counting rules of third-order SSE for high-energy external probes. Besides the singlenucleon current, we couple the photon field also to the meson-exchange currents of the twonucleon system. The calculations of [22, 27, 29] are strictly perturbative in the interaction kernel and therefore have the disadvantage that they become inapplicable below $\omega \sim 50$ $60 \mathrm{MeV}$, see discussion in Section 2.2.1. For example, they would over-predict the deuteron Thomson limit by more than a factor 2 [40]. This is the more damaging as the Thomson limit is a simple consequence of gauge invariance, so that the calculation in a too-simple application of Weinberg's counting rules obviously violates gauge invariance. The calculation presented in this publication does not suffer from that limitation. Instead, we implement Weinberg's original suggestion that chiral counting can safely only be applied to the twonucleon irreducible pieces of the amplitudes. The non-perturbative nature of two-nucleon rescattering must for energies well below the pion mass be implemented by iteration, no matter whether in initial, final or intermediate states. Since this approach reaches the exact Thomson limit, we are able to describe the low-energy $(\omega<60 \mathrm{MeV})$ data well.

It is worth noting that a fully self-consistent $\chi \mathrm{EFT}$ of the two-nucleon system is not yet available. To take the counting rules of the one-nucleon system simply over to the potential of the two-nucleon system was Weinberg's original proposal [30,31]. However, Nogga et al. and Birse [38, 39] showed recently that this approach is not self-consistent in $N N$-scattering, but that additional short-distance interactions are needed in higher partial waves to recover phase shifts which are insensitive to details of short-distance physics. While this problem does not affect the ${ }^{3} \mathrm{~S}_{1}$ and ${ }^{3} \mathrm{D}_{1}$ channels, it may be of importance in the $N N$-intermediate state. However, we use here a so-called "high-precision" $N N$ potential, AV18 [34], which reproduces the $N N$ partial waves reasonably well.

The various cross-checks we perform in this presentation make us confident that the cross sections and polarizabilities we obtain in this paper will be essentially unchanged once 
the fully systematic theory of few-nucleon systems with pion-nucleon interactions is found. Its relation to a more rigorous EFT approach will be elaborated on in a future publication on power-counting in two-nucleon $\chi$ EFT [41,43]. Weinberg's suggestion [30,31] of a hybrid approach seems still to be applicable in deuteron Compton scattering.

The dependence of deuteron Compton scattering on the nucleon polarizabilities has also been explored to next-to-next-to leading order in the EFT variant in which pions are integrated out [44 46]. While the Thomson limit is in this approach implemented automatically, the range of applicability of this "pion-less" EFT is limited to typical momenta well below the pion mass and thus to typical photon energies $\omega \lesssim m_{\pi}^{2} / M \approx 20 \mathrm{MeV}$. The available deuteron Compton data lie well above this scale.

Comparing to Refs. [20, 25, 26], we see the main advantage of our calculation in an improved treatment of the single-nucleon Compton multipoles, which in those works are only included via the leading [25, 26] and subleading [20] terms of a Taylor expansion in $\omega$, whereas we keep the full energy dependence of the Compton multipoles, including the explicit $\Delta(1232)$ following third-order SSE as worked out in [2]. The huge influence of the $\Delta(1232)$ in single-nucleon Compton backscattering is well-known. It is due to the strong paramagnetic $M 1$ coupling of the photon to the $N \rightarrow \Delta$ transition. A similarly strong influence of the $\Delta$ resonance has been found in deuteron Compton scattering in the backward direction in [22]. Therefore, we advocate to retain this degree of freedom explicitly in any Compton calculation.

This paper is organized as follows: In Section 2, we give a brief survey of the theoretical formalism applied, focusing on those diagrams which have a two-nucleon intermediate state. We close the section by demonstrating that our approach is gauge invariant and therefore fulfill the low-energy theorem [47, i.e. it generates the correct static limit. In Section 3, we discuss our predictions for four different photon energies, ranging from $50 \mathrm{MeV}$ to $100 \mathrm{MeV}$, and we compare those to data and to the $\mathcal{O}\left(\epsilon^{3}\right)$ SSE calculation of Ref. [22]. The subsequent Section 4 contains our fits of the isoscalar polarizabilities to all existing elastic deuteron Compton data. We show that the data are self-consistent and in good agreement with the theoretical expectation that isovector components are small. We conclude in Section 5, having shifted the most technical parts to Appendices $\mathrm{A}$ and $\mathrm{B}$ and to Ref. [40]. In Appendix C. we derive the total deuteron-photodisintegration cross section from our Compton amplitude in order to cross-check our calculation. We find perfect agreement with data and predictions from Effective Range Theory. We also published summaries of these findings previously in conference proceedings [42, 43].

\section{Theoretical Framework}

In this work, elastic deuteron Compton scattering is examined, including explicit $\Delta$ resonance degrees of freedom. In general, the $T$-matrix for Compton scattering off the deuteron is derived as the matrix element of the interaction kernel, evaluated between initial- and final-state deuteron wave functions, as explained in great detail in [27]:

$$
T=\left\langle\Psi_{f}\left|K_{\gamma \gamma}+K_{\gamma} G K_{\gamma}\right| \Psi_{i}\right\rangle
$$


The first piece in Eq. (2.1) is called the "two-nucleon irreducible" part and the second is the "two-nucleon reducible" part. Two-nucleon reducible diagrams are those which contain an intermediate state with only the two nucleons as particle content. $G$ is the two-particle Green's function, constructed from the two-nucleon irreducible interaction $V$ and the free two-nucleon Green's function. $K_{\gamma}$ denotes the coupling of one photon to the two-nucleon system, $K_{\gamma \gamma}$ is the two-nucleon irreducible kernel for the coupling of the two photons.

The main difference between this work and Ref. [22] is the treatment of those diagrams which are characterized by the propagation of the two nucleons in the intermediate state between the two photon interactions. In [22, 27, 29], such diagrams have been calculated following the power-counting rules of Effective Field Theory for "high-energy" external probes, i.e. $\omega \sim m_{\pi}$. For large photon energies, a perturbative treatment is possible as is easily understood heuristically: The absorption of a high-energy photon immediately tears the two nucleons apart, so the deuteron would be destroyed if the second photon was not emitted near-instantaneously. It turns out that up to leading-one-loop order in $\mathrm{HB} \chi \mathrm{PT}$, as well as in SSE, the only diagrams with a two-nucleon intermediate state are the nucleonpole diagrams, sketched in Fig. 1. Note that the $s$-channel diagram, i.e. the left diagram in Fig. 1, is part of $K_{\gamma} G K_{\gamma}$, as it has an intermediate state with only the two nucleons propagating. While the nucleons can in general interact with each other between the two photon vertices, the authors of Refs. [22, 27] calculated only up to leading-one-loop order, where no such interactions are possible. Therefore, we sketch the propagation of two free nucleons in Fig. 1 ,

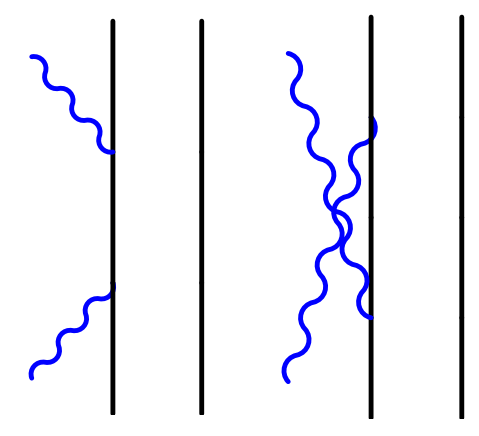

Figure 1: Nucleon-pole terms without rescattering of the nucleons between the two photon interactions. The photon-nucleon vertex is given by minimal substitution or magneticmoment interactions.

As a consequence of the power counting applied, the calculations of Refs. [22, 27 29] break down in the low-energy regime, manifested in an incorrect Thomson limit. Their lower energy limit was found to be of the order of 50-60 MeV. The reason for the mismatch at low energies is that one has to treat the $n p$-interaction non-perturbatively. Therefore, we now include the full $n p$ - $S$-matrix in the intermediate state. The possible rescattering between the two nucleons is denoted by a square in Fig. 2, where we sketch the differences between Ref. 222] and this work. Foremost, we do not only include the free propagation of the two nucleons in the nucleon-pole diagrams, Fig. 1, but we construct the full $n p$-Green's 


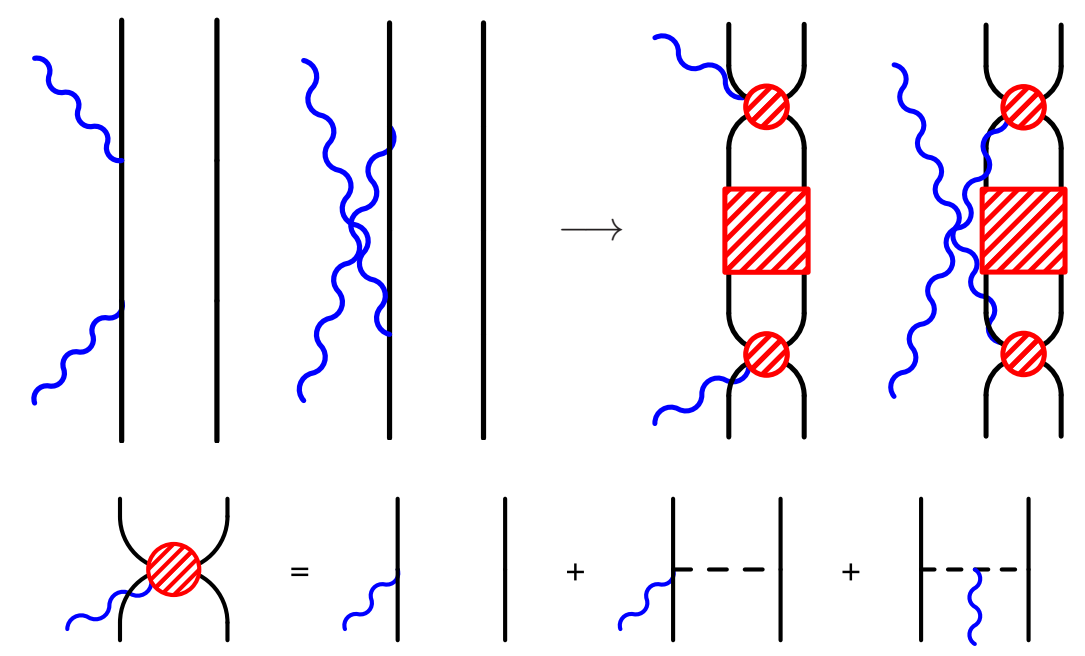

Figure 2: Upper line: Sketch of the different treatment of diagrams with two-nucleon intermediate state in the present work with respect to Refs. [22, 27]. The square symbolizes the $N N$-S-matrix. The blobs denote photon coupling to the one-body current and possible one-pion exchange, as indicated in the lower line.

function whenever a two-nucleon intermediate state is involved. Furthermore, we allow for more ways of coupling the photon field to the two nucleons with respect to Refs. [22, 27]: Besides coupling to the single-nucleon current, like in Fig. 1, we also include pion-exchange currents as shown in the lower line of Fig. 2, making use of Siegert's theorem [48, cf. Section 2.2. Such diagrams appear only at $\mathcal{O}\left(p^{4}\right)$ in $\mathrm{HB} \chi \mathrm{PT}$ and are therefore not included in [22]. In Ref. [28, 29], the $n p$-rescattering in the intermediate state is not fully included. It is well-known that only full inclusion of $n p$-rescattering, together with the appropriate explicit pion-exchange diagrams, generates the Thomson limit of deuteron Compton scattering [20, 25, 26, 49, 50] as direct consequence of only demanding gauge invariance [47].

In this publication, we follow closely the work of Karakowski and Miller [25, 26] to construct the rescattering and the photon coupling to the meson-exchange currents via Siegert's theorem, cf. Fig. 2. However, before we turn to the calculation of the diagrams with an intermediate $n p$-state, we recall all other contributions, which therefore are part of $K_{\gamma \gamma}$, see Eq. (2.1). As those terms have already been discussed in Ref. [22] and partly in earlier references therein, we shall be brief in the upcoming section.

\subsection{Diagrams without Intermediate $N N$-Scattering}

Except for the diagrams with two-nucleon intermediate states, see Fig. 2, we apply the power-counting rules of the Small Scale Expansion, where the expansion parameter is $\epsilon$, denoting either a small momentum, the pion mass or the mass difference $\Delta_{0}$ between the real part of the $\Delta$ mass and the nucleon mass. We refer the interested reader to [51] for the $N \pi$ Lagrangean and to [52] and [2] for the relevant pieces of the $\Delta \pi$ Lagrangean. The powercounting scheme that we use for the nucleon-structure part of our calculation is motivated 
by Weinberg's idea to count powers only in the interaction kernel [30, 31]. This hybrid approach is a well-established tool by now. While the kernel is power counted according to the rules of the Effective Field Theory, a deuteron wave function from state-of-the-art $N N$ potentials is used. Unfortunately, a fully self-consistent $\chi \mathrm{EFT}$ of the two-nucleon system is not yet available [38, 39]. We apply therefore here an extension of the hybrid approach which uses basic facts of nuclear phenomenology in the two-nucleon sector such as $n p$ rescattering and meson-exchange currents. Its relation to a more rigorous EFT approach will be given in a forthcoming work on power counting in two-nucleon $\chi$ EFT [41 43]. Still, the deuteron wave functions that we use are derived from modern $N N$-potentials: the AV18-potential [34] and the "NNLO chiral" potential [53]. This last potential also follows Weinberg's suggestion and is derived by applying $\mathrm{HB} \chi \mathrm{PT}$ power counting to the $N N$ potential $V$ itself. It therefore contains some terms which are strictly speaking of higher order. Except for the un-resolved power-counting issues mentioned in the Introduction, one does not expect this to be detrimental for the accuracy of our calculation. A NLO potential would actually suffice in our calculation since the photo-nuclear interaction kernel is expanded only up to NLO as well. We will demonstrate in Sec. 3.3 that the differences to a LO chiral potential are very small and can be used to estimate residual theoretical uncertainties.

Considering only contributions without intermediate two-nucleon states, there is another possibility to classify diagrams. It is the separation into one-body and two-body pieces, i.e. into diagrams where only one of the two nucleons or both of them are involved in the Compton-scattering process. Obviously, such a rigorous separation is no longer possible when we calculate the diagrams including $N N$-rescattering between the photon interactions, cf. Fig. 2, As in Ref. [22], we calculate up to $\mathcal{O}\left(\epsilon^{3}\right)$, i.e. there is no difference in the diagrams without an intermediate $n p$-state between this work and [22]. Therefore, we refer to Ref. [22] for further details on these contributions and only list again the various diagrams for completeness:

- One-body contributions without explicit $\Delta(1232)$ degrees of freedom. These are the single-nucleon seagull, Fig. 3(a), and the contributions from the leading chiral dynamics of the pion cloud around the nucleon (Figs. 3(c)-(f)). The pion pole (Fig. 3(b)), i.e. the $\pi^{0}$-exchange in the $t$-channel, does not contribute to deuteron Compton scattering at this order, as it is isovectorial. Note that the nucleon-pole terms, Fig. 1, also contribute at $\mathcal{O}\left(\epsilon^{3}\right)$ [22]. In this work, however, these diagrams are not only included perturbatively, like in Ref. [22, but we calculate them using the full $n p$-Green's function $G$, cf. Eq. (2.1). Their evaluation is postponed to Section 2.2.

- One-body diagrams with explicit $\Delta$ degrees of freedom, as shown in Fig. 4 . The $\Delta$-pole diagrams (Fig. $4(\mathrm{a})$ ) and the contributions from the pion cloud around the $\Delta(1232)$ (Figs. 4(b)-(e)).

- Two isoscalar short-distance one-body operators (Fig. 4(f)), whose coupling constants are denoted as $g_{117}, g_{118}$ in Table1. These operators, which we determine via fits to either proton or deuteron Compton cross-section data, contribute energy-independently 


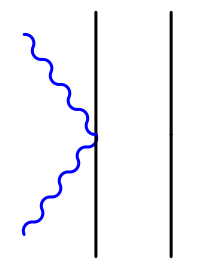

(a)

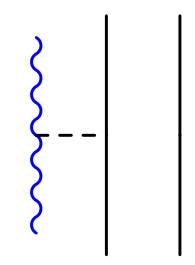

(b)

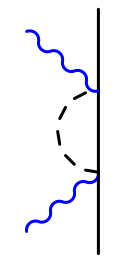

(c)

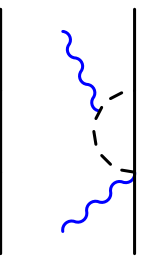

(d)

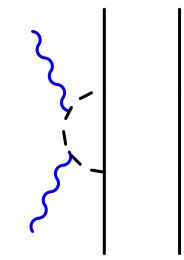

(e)

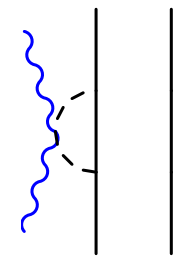

(f)

Figure 3: One-body interactions without $\Delta(1232)$ propagator contributing to deuteron Compton scattering up to $\mathcal{O}\left(\epsilon^{3}\right)$ in SSE. Permutations and crossed graphs are not shown.

to the dipole polarizabilities $\alpha_{E}^{s}$ and $\beta_{M}^{s}$. They are formally of $\mathcal{O}\left(\epsilon^{4}\right)$ but turn out to give an anomalously large contribution to the single-nucleon Compton amplitude. Therefore, we promote them to next-to-leading order as discussed in detail in [2]. As pointed out in Refs. [2,22], two isoscalar parameters suffice for an accurate description of all Compton data on the proton and deuteron. We will confirm later that isovectorial short-distance effects seem to be suppressed. We therefore do not augment the number of short-distance parameters which are promoted to lower orders beyond the necessary minimal set consisting of these two.

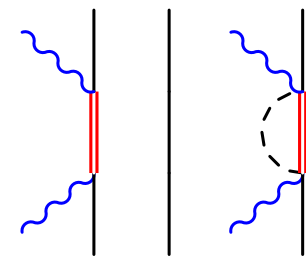

(a)

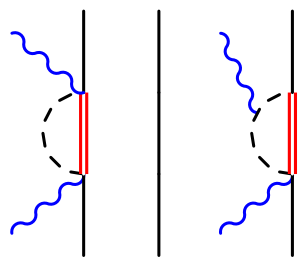

(b)

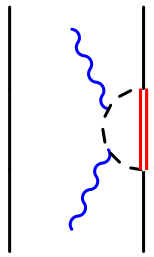

(c)

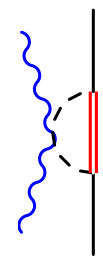

(d)

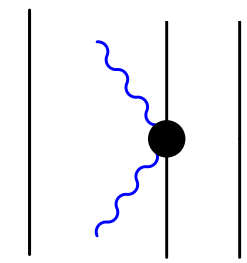

(e)

(f)

Figure 4: Additional one-body interactions which contribute to deuteron Compton scattering at $\mathcal{O}\left(\epsilon^{3}\right)$ in SSE compared to third-order $\mathrm{HB} \chi \mathrm{PT}$. Permutations and crossed graphs are not shown.

- Two-body contributions with one pion exchanged between the two nucleons (Fig. 5). As discussed in Ref. [22], the meson-exchange diagrams are identical in third-order $\mathrm{HB} \chi \mathrm{PT}$ and SSE. These diagrams, together with those given in Figs. 2 and 3(a) are responsible for complying with the low-energy theorem, as discussed in Section 2.2.4.

All these diagrams (Figs. 3 5) are included in our interaction kernel. The SSE singlenucleon amplitudes can be found in [2], while the two-body contributions are given explicitly in [27]. The only diagrams that remain to be calculated are the diagrams with an intermediate $n p$-state, cf. Fig. 2. 


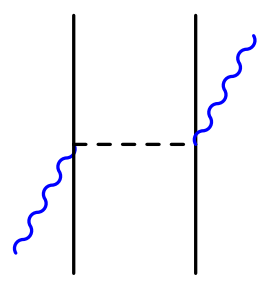

(a)

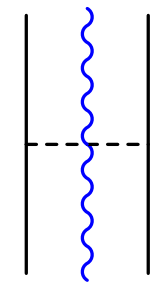

(b)

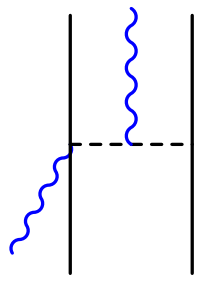

(c)

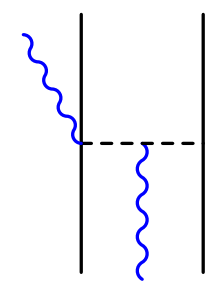

(d)

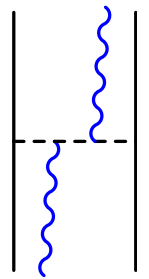

(e)

Figure 5: Two-body interactions contributing to the kernel for deuteron Compton scattering at $\mathcal{O}\left(\epsilon^{3}\right)$ in SSE. Diagrams which differ only by nucleon interchange are not shown.

\subsection{Diagrams with Intermediate $N N$-Scattering}

\subsubsection{A Short Note on the Thomson Limit}

Before we describe how we calculate the $N N$-rescattering diagrams sketched in Fig. 2, we consider the well-known Thomson limit, i.e. the deuteron Compton amplitude in the limit of vanishing photon energy:

$$
A_{d}^{\text {Thomson }}=\frac{e^{2}}{m_{d}} \hat{\epsilon}_{\lambda_{i}} \cdot \hat{\epsilon}_{\lambda_{f}}^{*} \approx \frac{e^{2}}{2 m_{N}} \hat{\epsilon}_{\lambda_{i}} \cdot \hat{\epsilon}_{\lambda_{f}}^{*} .
$$

Friar showed that Eq. (2.2) is a consequence of current conservation and - as long as there are no photons in internal loops - gauge invariance [47]. The EFT calculations [22, 27, 29] investigate the régime $\omega \sim m_{\pi}$ and thus are by construction inapplicable in that limit. If taken at face-value, they would there also violate gauge invariance, albeit their interaction kernel is gauge invariant by construction. The violation appears when evaluating the kernel between the deuteron wave functions, without allowing the two nucleons in the intermediate state to interact with each other. The reason is that the deuteron wave function implies this interaction, which can be interpreted as the exchange of mesons, e.g. of pions, between the two nucleons. In order to achieve current conservation and gauge invariance, it is therefore mandatory to include rescattering of the two nucleons on one hand and to couple the photons to these meson-exchange currents, cf. Fig. 2 and Refs. [20, 25, 26]. It is one of the main advantages of this work with respect to [22, 27 29] that our calculation does fulfill Eq. (2.2).

Reaching the Thomson limit is a non-trivial check because the deuteron mass is involved, whereas the Thomson seagull for Compton scattering from the proton, Fig. 3(a), yields

$$
A_{p}^{\text {Thomson }}=\frac{e^{2}}{m_{p}} \hat{\epsilon}_{\lambda_{i}} \cdot \hat{\epsilon}_{\lambda_{f}}^{*}
$$

with $m_{p}$ the proton mass. The single-neutron amplitude vanishes in the static limit. Therefore, all other contributions to deuteron Compton scattering in the limit $\omega \rightarrow 0$ have to cancel half of the proton amplitude (2.3). In Section 2.2.4 we discuss how this cancellation comes about, after sketching in Sections 2.2.2 and 2.2.3 our way to calculate diagrams with intermediate $n p$-scattering. 


\subsubsection{Dominant Terms}

In the following, we briefly explain the "Green's-function method" to include the $N N$ rescattering in the diagrams given in Fig. 2, using second-order time-ordered perturbation theory in the two-photon interaction. For further details see Appendix B] and Refs. [25, 26, 40]. In general, the scattering amplitude for these processes can be written as

$$
\begin{aligned}
\mathcal{M}_{f i} & =\sum_{C}\left\{\frac{\left\langle d_{f}, \gamma_{f}\left|H^{\mathrm{int}}\right| C\right\rangle\left\langle C\left|H^{\mathrm{int}}\right| d_{i}, \gamma_{i}\right\rangle}{\omega+\frac{\omega^{2}}{2 m_{d}}-B-E_{C}}\right. \\
& \left.+\frac{\left\langle d_{f}, \gamma_{f}\left|H^{\mathrm{int}}\right| C, \gamma_{f}, \gamma_{i}\right\rangle\left\langle C, \gamma_{f}, \gamma_{i}\left|H^{\mathrm{int}}\right| d_{i}, \gamma_{i}\right\rangle}{-\omega+\frac{\omega^{2}}{2 m_{d}}-B-E_{C}-\frac{\vec{P}_{C}^{2}}{2 m_{C}}}\right\},
\end{aligned}
$$

where we sum over all possible intermediate eigenstates $C$ of the $N N$-interaction Hamiltonian. Recall that the intermediate rescattering state (2.4) is the off-shell $S$-matrix of $N N$-scattering, as pointed out at the beginning of Sec. 2. It contains also the case that the two nucleons do not interact with each other between photon emission and absorption.

The two terms in Eq. (2.4) correspond to the two diagrams shown on the right hand side of Fig. 2. We sketch these diagrams once again in Fig. 6 in order to explain the various terms in the denominators of Eq. (2.4), constituting the energy of the intermediate nucleons. In the $\gamma d \mathrm{~cm}$ frame, the incoming and outgoing photons have the same energy $\omega . B$ denotes
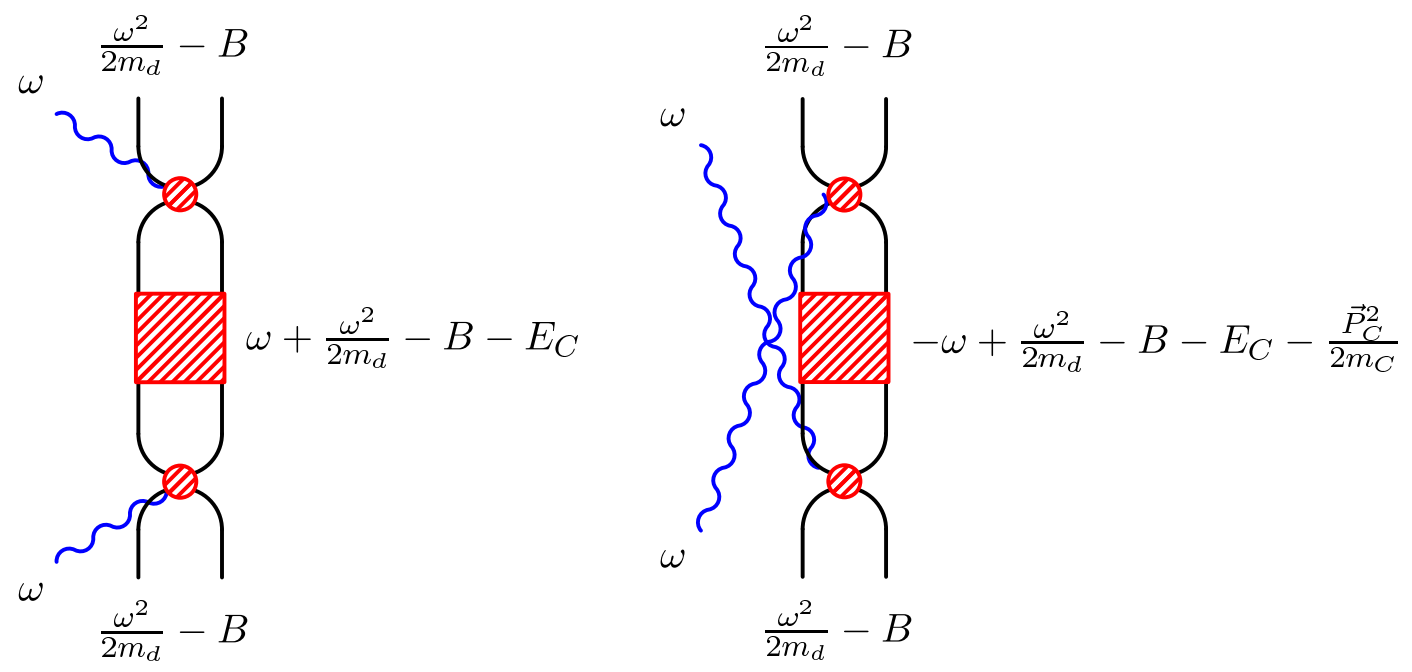

Figure 6: Diagrams with two-nucleon intermediate states (hatched square) in the $s$-channel (left) and in the $u$-channel (right). The labels denote the energies of the various particles and states.

the deuteron binding energy, $\frac{\omega^{2}}{2 m_{d}}$ is the kinetic energy of the incoming deuteron, $\frac{\vec{P}_{C}^{2}}{2 m_{C}}$ that of the intermediate two-nucleon system. For our numerical evaluations we use the masses $m_{C}=2 m_{N}, m_{d}=2 m_{N}-B$ and neglect isospin-breaking effects, $m_{p} \equiv m_{n} \equiv m_{N}$. As we 
calculate in the cm frame of the $\gamma d$ system, $\frac{\vec{P}_{C}^{2}}{2 m_{C}}=0$ in the $s$-channel diagram, whereas $\vec{P}_{C}=-\vec{k}_{i}-\vec{k}_{f}$ in the $u$-channel, i.e. $\frac{\vec{P}_{C}^{2}}{2 m_{C}}=\frac{\omega^{2}}{m_{C}}(1+\cos \theta) .-E_{C}$ denotes the excitation energy of the intermediate state $C$.

The interaction Hamiltonian in Eq. (2.4) is

$$
H^{\mathrm{int}}=-\int \vec{J}(\vec{\xi}) \cdot \vec{A}(\vec{\xi}) d^{3} \xi
$$

An explicit expression for the photon field, expanded into multipoles, has been derived in [25, 26] in analogy to Ref. [54] and is given in Eq. (A.1). It consists of three parts and can schematically be written as

$$
\vec{A}=\vec{\nabla} \phi+\vec{A}^{(1)}+\vec{A}^{(2)}
$$

with $\overrightarrow{A^{(1)}}$ denoting the magnetic part of $\vec{A}, \vec{\nabla} \phi+\vec{A}^{(2)}$ the electric part. The definitions of the function $\phi(\vec{\xi})$, as well as of $\vec{A}^{(1)}$ and $\vec{A}^{(2)}$, are given in Appendix A. Now we systematically replace the photon field in the interaction Hamiltonian (2.5) by the three terms contained in Eq. (2.6). Therefore, when we only distinguish between the various possibilities for $\vec{A}$, we find nine different combinations in Eq. (2.4). The largest contributions are those where we substitute $\vec{A}(\vec{\xi}) \rightarrow \vec{\nabla} \phi(\vec{\xi})$ at both vertices, which is the only part of the photon field that contributes for $\omega=0$, cf. Appendix A. Further terms, where this replacement is made only once, are discussed in Section 2.2.3. Only a few combinations of interactions without the gradient part of $\vec{A}$ give visible contributions. These are also taken care of in Section 2.2.3.

Contributions to the elastic cross sections of the order of $\leq 2 \%$ are neglected throughout this article since the theoretical uncertainty from effects which are higher than $\mathcal{O}\left(\epsilon^{3}\right)$ is larger than that. This is discussed and quantified in detail in the following Sections and summarized in the Conclusions. Most of these contributions are treated in detail in Hildebrandt's PhD thesis [40]. The numerical error is $\leq 2 \%$ at very low energies and less for $\omega \sim m_{\pi}$, see e.g. the discussion of the Thomson limit in Sect. 2.2.4.

In this section, we calculate Eq. (2.4) with $H^{\text {int }} \rightarrow-\int \vec{J}(\vec{\xi}) \cdot \vec{\nabla} \phi(\vec{\xi}) d^{3} \xi$ simultaneously at both vertices, i.e. we restrict ourselves to the terms arising from minimal coupling. In order to simplify the calculation on the one hand, and to ensure gauge invariance and the correct Thomson limit on the other, we integrate by parts and use current conservation:

$$
\begin{aligned}
-\int \vec{J}(\vec{\xi}) \cdot \vec{\nabla} \phi(\vec{\xi}) d^{3} \xi & =\int \vec{\nabla} \cdot \vec{J}(\vec{\xi}) \phi(\vec{\xi}) d^{3} \xi \\
\vec{\nabla} \cdot \vec{J}(\vec{\xi}) & =-\frac{\partial \rho(\vec{\xi})}{\partial t}=-i[H, \rho(\vec{\xi})]
\end{aligned}
$$

The fact that one only needs to know the charge density $\rho$ in order to calculate the amplitude in the long-wavelength limit is referred to as "Siegert's theorem" [48]. For $\rho(\vec{\xi})$ one can find in [55] the general decomposition

$$
\rho(\vec{\xi})=\rho^{(0)}(\vec{\xi})+\rho^{\mathrm{ex}}\left(\vec{\xi} ; \vec{x}_{p}, \vec{x}_{n}\right)
$$


with $\rho^{(0)}$ the charge density of the two nucleons and $\vec{x}_{p}, \vec{x}_{n}$ the position of proton and neutron, respectively. $\rho^{\text {ex }}\left(\vec{\xi} ; \vec{x}_{p}, \vec{x}_{n}\right)$ is the charge density arising from meson-exchange currents, e.g. from those given in the lower line of Fig. 2. The dominant term in Eq. (2.9) is

$$
\rho^{(0)}(\vec{\xi})=\sum_{j=n, p} e_{j} \delta\left(\vec{\xi}-\vec{x}_{j}\right)=e \delta\left(\vec{\xi}-\vec{x}_{p}\right)
$$

which is the only non-vanishing contribution to $\rho(\vec{\xi})$ in the static limit ("Siegert's hypothesis" [48]). Note that the $\delta$-functions in Eq. (2.10) indicate that the two nucleons are treated as pointlike particles, i.e. unlike the authors of e.g. Ref. [20] we do not introduce any nucleon form factors. We also performed calculations including $\rho^{\operatorname{ex}}\left(\vec{\xi} ; \vec{x}_{p}, \vec{x}_{n}\right)$. From these investigations, which are reported in Ref. [40], we conclude that $\rho^{\text {ex }}$ is well negligible in the energy range considered. Indeed, such terms are suppressed by three orders in the EFT power-counting, as shown in Refs. [36, 56]. Therefore, we are only concerned with $\rho^{(0)}(\vec{\xi})$.

Albeit it is not obvious, the use of current conservation in Eq. (2.8) causes that mesonexchange currents (cf. Fig. 7) are also implicitly included in the calculation, as

$$
\vec{\nabla} \cdot \vec{J}^{\mathrm{ex}}=-i\left[V^{\mathrm{ex}} \vec{\tau}_{1} \cdot \vec{\tau}_{2}, \rho^{(0)}\right]-i\left[H, \rho^{\mathrm{ex}}\right] .
$$

$V^{\mathrm{ex}}$ is the $n p$-potential from one-pion exchange [55], which is part of the Hamiltonian $H$. $\vec{\tau}_{i}$ is the isospin operator of the $i$ th nucleon. These contributions, which go beyond coupling the photon field to the single-nucleon current, like in Fig. 1, were already indicated in Fig. 2,

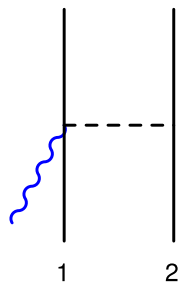

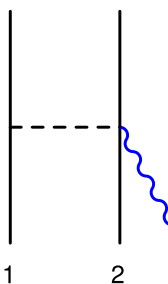

(a)

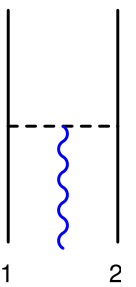

(b)

Figure 7: One-pion-exchange currents contributing to our calculation: the "Kroll-Ruderman current" (a) and the "pion-pole current" (b).

Substituting $\vec{\nabla} \cdot \vec{J}$ by $-i\left[H, \rho^{(0)}(\vec{\xi})\right]$ in Eq. (2.7), the integral over the dummy variable $\vec{\xi}$ can easily be performed to yield

$$
H^{\mathrm{int}}=-\int \vec{J}(\vec{\xi}) \cdot \vec{\nabla} \phi(\vec{\xi}) d^{3} \xi=-i\left[H, e \phi\left(\vec{x}_{p}\right)\right],
$$

where $H$ is the full Hamiltonian of the $n p$-system

$$
H=\frac{\vec{p}_{p}^{2}}{2 m_{p}}+\frac{\vec{p}_{n}^{2}}{2 m_{n}}+V
$$


with the $n p$-potential $V$. To evaluate the commutator (2.12), we switch to $\mathrm{cm}$ variables, i.e.

$$
\vec{p}=\frac{\vec{p}_{p}-\vec{p}_{n}}{2}, \quad \vec{P}=\vec{p}_{p}+\vec{p}_{n}, \quad \vec{r}=\vec{x}_{p}-\vec{x}_{n}, \quad \vec{R}=\frac{\vec{x}_{p}+\vec{x}_{n}}{2} .
$$

Our analysis shows that recoil corrections, which arise from the cm motion of the deuteron, are well negligible. This observation agrees with Ref. [25, 26], where such corrections have been evaluated as well. The net effect is that we may set $\vec{R}=\overrightarrow{0}$, i.e. we neglect the $\mathrm{cm}$ velocity of the two nucleons. As a consequence, we find $\vec{x}_{p}=\vec{r} / 2$ and the Hamiltonian (2.13) simplifies to the "internal" Hamiltonian

$$
H^{n p}=\frac{\vec{p}^{2}}{m_{N}}+V
$$

Therefore, we can rewrite Eq. (2.12) as

$$
H^{\text {int }}=-i\left[H^{n p}, e \phi(\vec{r} / 2)\right] .
$$

Inserting the commutator (2.16) into Eq. (2.4) and defining $\hat{\phi}_{i}=e \phi_{i}(\vec{r} / 2), \hat{\phi}_{f}=e \phi_{f}(\vec{r} / 2)$ in analogy to Ref. [25,26] we get

$$
\begin{aligned}
\mathcal{M}_{f i}^{\phi \phi} & =-\sum_{C}\left\{\frac{\left\langle d_{f}\left|\left[H^{n p}, \hat{\phi}_{f}\right]\right| C\right\rangle\left\langle C\left|\left[H^{n p}, \hat{\phi}_{i}\right]\right| d_{i}\right\rangle}{\omega+\frac{\omega^{2}}{2 m_{d}}-B-E_{C}}\right. \\
& \left.+\frac{\left\langle d_{f}\left|\left[H^{n p}, \hat{\phi}_{i}\right]\right| C\right\rangle\left\langle C\left|\left[H^{n p}, \hat{\phi}_{f}\right]\right| d_{i}\right\rangle}{-\omega+\frac{\omega^{2}}{2 m_{d}}-B-E_{C}-\frac{\vec{P}_{C}^{2}}{2 m_{C}}}\right\} .
\end{aligned}
$$

In order to keep track of the various combinations of interaction Hamiltonians we have labeled the double- $\phi$ transition matrix ' $\phi \phi$ '; the photon states have been skipped for brevity. Now the commutators are expanded and, as $\left|d_{i, f}\right\rangle,|C\rangle$ are eigenstates of $H^{n p}$, we can act with $H^{n p}$ on these states. We end up with four amplitudes, which have already been derived (in the lab system) in Ref. [25, 26]:

$$
\begin{aligned}
\mathcal{M}_{f i}^{\phi \phi 1} & =\left(\omega+\frac{\omega^{2}}{2 m_{d}}\right)^{2} \sum_{C} \frac{\left\langle d_{f}\left|\hat{\phi}_{f}\right| C\right\rangle\left\langle C\left|\hat{\phi}_{i}\right| d_{i}\right\rangle}{\omega+\frac{\omega^{2}}{2 m_{d}}-B-E_{C}} \\
\mathcal{M}_{f i}^{\phi \phi 2} & =\left(\omega+\frac{\vec{P}_{C}^{2}}{2 m_{C}}-\frac{\omega^{2}}{2 m_{d}}\right)^{2} \sum_{C} \frac{\left\langle d_{f}\left|\hat{\phi}_{i}\right| C\right\rangle\left\langle C\left|\hat{\phi}_{f}\right| d_{i}\right\rangle}{-\omega-\frac{\vec{P}_{C}^{2}}{2 m_{C}}+\frac{\omega^{2}}{2 m_{d}}-B-E_{C}} \\
\mathcal{M}_{f i}^{\phi \phi 3} & =\left(\frac{\vec{P}_{C}^{2}}{2 m_{d}}-\frac{\omega^{2}}{m_{d}}\right)\left\langle d_{f}\left|\hat{\phi}_{f} \hat{\phi}_{i}\right| d_{i}\right\rangle \\
\mathcal{M}_{f i}^{\phi \phi 4} & =\frac{1}{2}\left\langle d_{f}\left|\left[\left[H^{n p}, \hat{\phi}_{i}\right], \hat{\phi}_{f}\right]+\left[\left[H^{n p}, \hat{\phi}_{f}\right], \hat{\phi}_{i}\right]\right| d_{i}\right\rangle
\end{aligned}
$$

\footnotetext{
${ }^{1}$ There is one exception to this rule: We do include the kinetic energy of the two-nucleon system in energy denominators, cf. Eq. (2.4).
} 
$\mathcal{M}_{f i}^{\phi \phi 4}$ is the only one of these amplitudes which contributes in the static limit. It is responsible for the correct low-energy behavior of the calculation, as will be discussed in detail in Section 2.2.4.

We defer the evaluation of these amplitudes to Appendix $B$ and turn now to those contributions, where the substitution $\vec{A}(\vec{\xi}) \rightarrow \vec{\nabla} \phi(\vec{\xi})$ (cf. the beginning of this section) is made at most once.

\subsubsection{Subleading Terms}

So far we only considered contributions arising from minimal coupling of the photon field to the two-nucleon system at both vertices. In the following, we describe how to calculate the amplitudes given in Eq. (2.4), when the replacement

$$
H^{\mathrm{int}}=-\int \vec{J}(\vec{\xi}) \cdot \vec{A}(\vec{\xi}) d^{3} \xi \rightarrow-\int \vec{J}(\vec{\xi}) \cdot \vec{\nabla} \phi(\vec{\xi}) d^{3} \xi
$$

is made only once. The term 'subleading' refers to the fact that the resulting amplitude is numerically less important than that of Section 2.2.2 - its contribution to the differential cross section is suppressed with respect to the dominant terms from Section 2.2 .2 by at least one order of magnitude for all energies and angles considered, see Fig. 11, The amplitude is denoted by $\mathcal{M}_{f i}^{\phi}$ and follows immediately from Eq. (2.4):

$$
\begin{aligned}
\mathcal{M}_{f i}^{\phi} & =\sum_{C}\left\{\frac{\left\langle d_{f}\left|\int \vec{J}(\vec{\xi}) \cdot \vec{\nabla} \phi_{f}(\vec{\xi}) d^{3} \xi\right| C\right\rangle\left\langle C\left|\int \vec{J}(\vec{\xi}) \cdot \vec{A}(\vec{\xi}) d^{3} \xi\right| d_{i}\right\rangle}{\omega+\frac{\omega^{2}}{2 m_{d}}-B-E_{C}}\right. \\
& +\frac{\left\langle d_{f}\left|\int \vec{J}(\vec{\xi}) \cdot \vec{A}(\vec{\xi}) d^{3} \xi\right| C\right\rangle\left\langle C\left|\int \vec{J}(\vec{\xi}) \cdot \vec{\nabla} \phi_{i}(\vec{\xi}) d^{3} \xi\right| d_{i}\right\rangle}{\omega+\frac{\omega^{2}}{2 m_{d}}-B-E_{C}} \\
& +\frac{\left\langle d_{f}\left|\int \vec{J}(\vec{\xi}) \cdot \vec{\nabla} \phi_{i}(\vec{\xi}) d^{3} \xi\right| C\right\rangle\left\langle C\left|\int \vec{J}(\vec{\xi}) \cdot \vec{A}(\vec{\xi}) d^{3} \xi\right| d_{i}\right\rangle}{-\omega+\frac{\omega^{2}}{2 m_{d}}-\frac{\vec{P}_{C}^{2}}{2 m_{C}}-B-E_{C}} \\
& \left.+\frac{\left\langle d_{f}\left|\int \vec{J}(\vec{\xi}) \cdot \vec{A}(\vec{\xi}) d^{3} \xi\right| C\right\rangle\left\langle C\left|\int \vec{J}(\vec{\xi}) \cdot \vec{\nabla} \phi_{f}(\vec{\xi}) d^{3} \xi\right| d_{i}\right\rangle}{-\omega+\frac{\omega^{2}}{2 m_{d}}-\frac{\vec{P}_{C}^{2}}{2 m_{C}}-B-E_{C}}\right\}
\end{aligned}
$$

Now we perform the same steps as described in Eqs. (2.12-2.21), i.e. we first replace $\int \vec{J}(\vec{\xi})$. $\vec{\nabla} \phi(\vec{\xi}) d^{3} \xi$ by $i\left[H^{n p}, e \phi(\vec{r} / 2)\right]$, then act with $H^{n p}$ on $|d\rangle$ and $|C\rangle$, respectively, and finally add and subtract terms in order to perform some cancellations against the denominator. 
We find, again neglecting recoil terms and the deuteron velocity,

$$
\begin{aligned}
\mathcal{M}_{f i}^{\phi} & =i \sum_{C}\left\{\left\langle d_{f}\left|\hat{\phi}_{f}\right| C\right\rangle\left\langle C\left|\int \vec{J}(\vec{\xi}) \cdot \vec{A}(\vec{\xi}) d^{3} \xi\right| d_{i}\right\rangle\right. \\
& -\left(\omega+\frac{\omega^{2}}{2 m_{d}}\right) \frac{\left\langle d_{f}\left|\hat{\phi}_{f}\right| C\right\rangle\left\langle C\left|\int \vec{J}(\vec{\xi}) \cdot \vec{A}(\vec{\xi}) d^{3} \xi\right| d_{i}\right\rangle}{\omega+\frac{\omega^{2}}{2 m_{d}}-B-E_{C}} \\
& -\left\langle d_{f}\left|\int \vec{J}(\vec{\xi}) \cdot \vec{A}(\vec{\xi}) d^{3} \xi\right| C\right\rangle\left\langle C\left|\hat{\phi}_{i}\right| d_{i}\right\rangle \\
& +\left(\omega+\frac{\omega^{2}}{2 m_{d}}\right) \frac{\left\langle d_{f}\left|\int \vec{J}(\vec{\xi}) \cdot \vec{A}(\vec{\xi}) d^{3} \xi\right| C\right\rangle\left\langle C\left|\hat{\phi}_{i}\right| d_{i}\right\rangle}{\omega+\frac{\omega^{2}}{2 m_{d}}-B-E_{C}} \\
& +\left\langle d_{f}\left|\hat{\phi}_{i}\right| C\right\rangle\left\langle C\left|\int \vec{J}(\vec{\xi}) \cdot \vec{A}(\vec{\xi}) d^{3} \xi\right| d_{i}\right\rangle \\
& +\left(\omega-\frac{\omega^{2}}{2 m_{d}}+\frac{\vec{P}_{C}^{2}}{2 m_{C}}\right) \frac{\left\langle d_{f}\left|\hat{\phi}_{i}\right| C\right\rangle\left\langle C\left|\int \vec{J}(\vec{\xi}) \cdot \vec{A}(\vec{\xi}) d^{3} \xi\right| d_{i}\right\rangle}{-\omega+\frac{\omega^{2}}{2 m_{d}}-\frac{\vec{P}_{C}^{2}}{2 m_{C}}-B-E_{C}} \\
& -\left\langle d_{f}\left|\int \vec{J}(\vec{\xi}) \cdot \vec{A}(\vec{\xi}) d^{3} \xi\right| C\right\rangle\left\langle C\left|\hat{\phi}_{f}\right| d_{i}\right\rangle \\
& \left.-\left(\omega-\frac{\omega^{2}}{2 m_{d}}+\frac{\vec{P}_{C}^{2}}{2 m_{C}}\right) \frac{\left\langle d_{f}\left|\int \vec{J}(\vec{\xi}) \cdot \vec{A}(\vec{\xi}) d^{3} \xi\right| C\right\rangle\left\langle C\left|\hat{\phi}_{f}\right| d_{i}\right\rangle}{-\omega+\frac{\omega^{2}}{2 m_{d}}-\frac{\vec{P}_{C}^{2}}{2 m_{C}}-B-E_{C}}\right\} .
\end{aligned}
$$

Whenever the energy denominator has been canceled, the sum over $C$ may be collapsed. As $\hat{\phi}\left(\int \vec{J}(\vec{\xi}) \cdot \vec{A}(\vec{\xi}) d^{3} \xi\right)=\left(\int \vec{J}(\vec{\xi}) \cdot \vec{A}(\vec{\xi}) d^{3} \xi\right) \hat{\phi}$, these four terms cancel exactly, and only the terms including an energy denominator remain.

The relevant parts of the photon field $\vec{A}(\vec{\xi})$ are the non-gradient terms in Eq. (2.6). The current $\vec{J}(\vec{\xi})$ includes one-body and two-body currents. The latter are the pion-exchange currents of Fig. 7, however we found that only the Kroll-Ruderman current, Fig. 7)(a), gives visible contributions. The single-nucleon current consists of two parts, which we call $\vec{J}^{(\sigma)}$ and $\vec{J}(p)$, with

$$
\begin{aligned}
& \vec{J}^{(\sigma)}(\vec{\xi})=\frac{e}{2 m_{N}} \sum_{j=n, p}\left[\vec{\nabla}_{\xi} \times \mu_{j} \vec{\sigma}_{j} \delta\left(\vec{\xi}-\vec{x}_{j}\right)\right], \\
& \vec{J}^{(p)}(\vec{\xi})=\frac{1}{2 m_{N}} \sum_{j=n, p}\left\{e_{j} \delta\left(\vec{\xi}-\vec{x}_{j}\right), \vec{p}_{j}\right\},
\end{aligned}
$$

cf. e.g. [55]. So we may schematically write

$$
\vec{J}(\vec{\xi})=\vec{J}^{(\sigma)}(\vec{\xi})+\vec{J}^{(p)}(\vec{\xi})+\vec{J}^{\mathrm{KR}}(\vec{\xi})
$$

$\mu_{j}$ is the magnetic moment, $\vec{\sigma}_{j}$ the spin operator and $\vec{p}_{j}$ the momentum of the $j$ th nucleon. We observe sizeable contributions only from the magnetic moment interaction encoded in 
the spin current $\vec{J}^{(\sigma)}$, Eq. (2.25), whereas $\vec{J}^{(p)}$ turned out negligibly small. Our notation for the amplitudes is $\mathcal{M}_{f i}^{\phi \sigma}$ when we replace $\vec{J}(\vec{\xi})$ by $\vec{J}^{(\sigma)}(\vec{\xi})$ in Eq. (2.24) and $\mathcal{M}_{f i}^{\phi \mathrm{KR}}$ when we use the Kroll-Ruderman current instead. As this part is rather technical, we shift it to Appendix B. There, we also discuss those amplitudes which do not contain the gradient part of the photon field but nevertheless give sizeable contributions, i.e. $\mathcal{M}_{f i}^{\sigma \sigma}$ and $\mathcal{M}_{f i}^{\mathrm{KR} \sigma}$.

Some of these contributions have been considered before, namely diagrams with one photon coupling to one-pion-exchange and one photon coupling to a nucleon, see [28] and [29, Fig. 9]. As demonstrated there, they extend the region of validity $\omega \sim m_{\pi}$ of the kernel "without" rescattering to lower energies, but are not sufficient to restore the Thomson limit.

Two-body currents with explicit $\Delta(1232)$ degrees of freedom, as displayed in Fig. 8, are suppressed by one order in $\epsilon$ with respect to the Kroll-Ruderman current, Fig. 7(a), due to the $\gamma N \Delta$ vertex being part of $\mathcal{L}_{N \Delta}^{(2)}$ [5, 6]. This agrees with the findings of [20], where such contributions to elastic deuteron Compton scattering below $100 \mathrm{MeV}$ were claimed to be of the order of $2 \%$. A similar size is reported in [55] for the process $n p \rightarrow d \gamma$, where the contributions from the $\Delta(1232)$ current turn out to be considerably smaller than those from pionic exchange currents. Therefore, and due to the excellent agreement of the total deuteron-photodisintegration cross section, extracted from our elastic Compton amplitude, with data, cf. Appendix C, we so far refrain from including these terms into our calculation. It would be an interesting future task to perform a detailed investigation of their size.
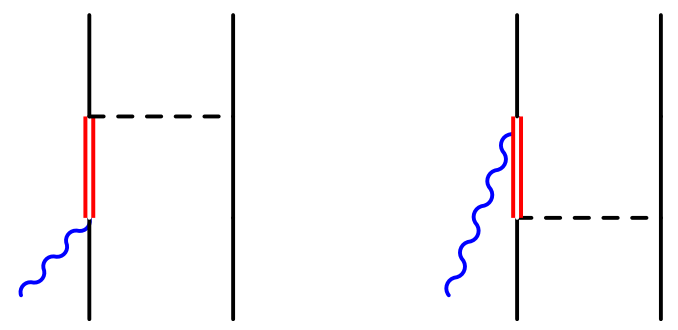

Figure 8: Exemplary one-pion-exchange currents with explicit $\Delta(1232)$ degrees of freedom.

Now we have prepared all ingredients of our deuteron Compton calculation. In the next section we demonstrate that it fulfills the well-known low-energy theorem, Eq. (2.2), i.e. we obtain the correct low-energy limit within our approach. In Section 3 , we present our results at non-zero energies and compare them to those from Ref. [22] and to data.

\subsubsection{Low-Energy (Thomson) Limit}

This work constitutes an extended hybrid approach to the calculations of Refs. [22, 27, 29] which are not applicable for photon energies below $50 \mathrm{MeV}$. In this section, we prove that we have indeed removed the limitations of these works at low energies, i.e. we reach the correct limit of vanishing photon energy, Eq. (2.2).

The only non-vanishing amplitudes in the static limit - except for the proton seagull, Fig. 3. (a) - are the explicit pion-exchange diagrams, Fig. 5, and the double-commutator term, 
Eq. (2.21). This double-commutator involves the internal Hamiltonian $H^{n p}=\frac{\vec{p}^{2}}{m_{N}}+V$, cf. Eq. (2.15), and therefore can be separated into a kinetic and a potential part. Arenhövel showed analytically that in the static limit, the potential energy part, using the one-pionexchange potential, cancels exactly the contributions from explicit pion exchange, Fig. 5] [49]. Therefore, the kinetic energy part has to cancel half of the proton seagull. This can easily be shown to be true, cf. Ref. [25, 26] or [40]. Note that the Thomson amplitude (2.2) is independent of the deuteron wave function and the $n p$-potential chosen.

Our numerical evaluation agrees well with the Thomson limit (2.2), as demonstrated in a comparison (Fig. 9) between the proton Compton cross section, $\left(\frac{1}{2}\right)^{2}=\frac{1}{4}$ of this cross section and the deuteron Compton cross section at zero photon energy (we remind the reader that $\left.\frac{d \sigma}{d \Omega} \propto\left|\mathcal{M}_{f i}\right|^{2}\right)$. The latter two curves are nearly indistinguishable. In the right panel of Fig. 9, we see that the relative error $\left(\frac{d \sigma}{d \Omega}\right)_{d} /\left(\frac{1}{4} \frac{d \sigma}{d \Omega}\right)_{p}-1$ is constant in $\theta$ and less than $2 \%$. Therefore it can be accounted for by a constant factor. The main part of this discrepancy is due to numerical uncertainties in the normalization of the wave function within our code.
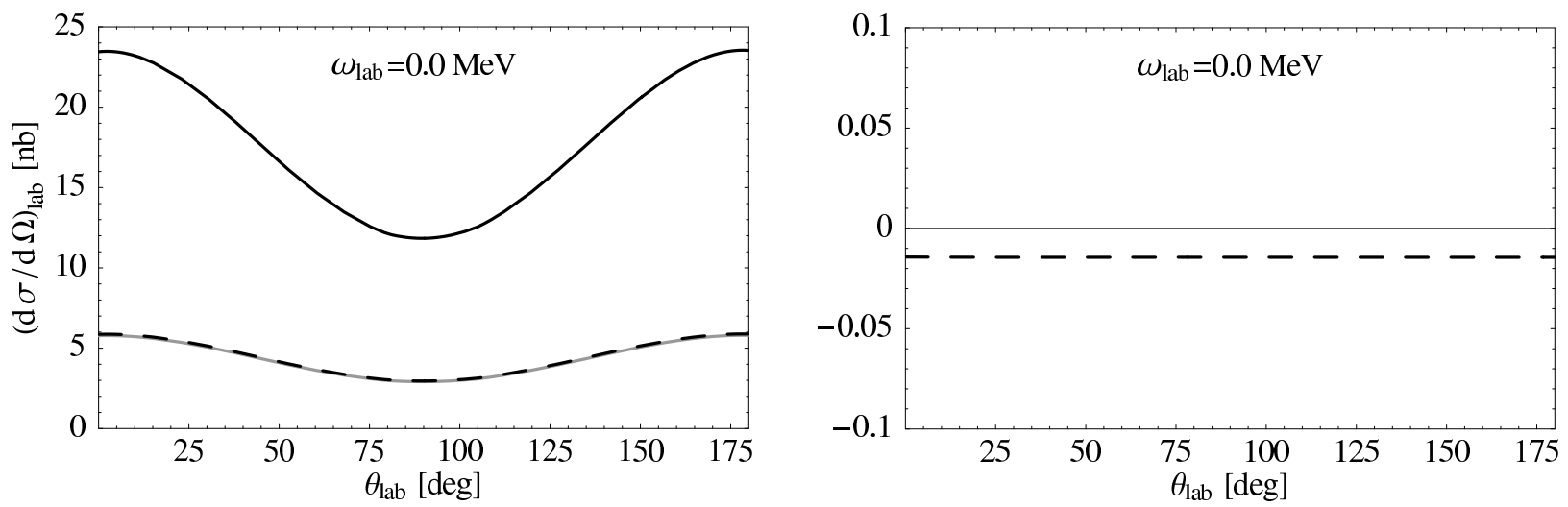

Figure 9: Left panel: Comparison of the proton (black, solid), deuteron (grey, solid) and $1 / 4$ of the proton (black, dashed) Compton cross section in the static limit. The function plotted in the right panel is $\left(\frac{d \sigma}{d \Omega}\right)_{d} /\left(\frac{1}{4} \frac{d \sigma}{d \Omega}\right)_{p}-1$.

In this section, we showed that our Green's-function hybrid approach to deuteron Compton scattering fulfills the low-energy theorem and therefore guarantees gauge invariance of the calculation. In the next section, we present our results for non-zero photon energies. From the good description of all data available we conclude that we have achieved a consistent description of $\gamma d$ scattering for photon energies ranging from $0 \mathrm{MeV}$ up to $\omega \sim 100 \mathrm{MeV}$.

\section{Predictions and Discussion}

\subsection{Comparison to Previous Work}

In Fig. 10, we show our predictions for the elastic deuteron Compton cross sections, compared to those from Ref. [22] and to the data from Illinois [7], Lund [8] and SAL [9]. Our 

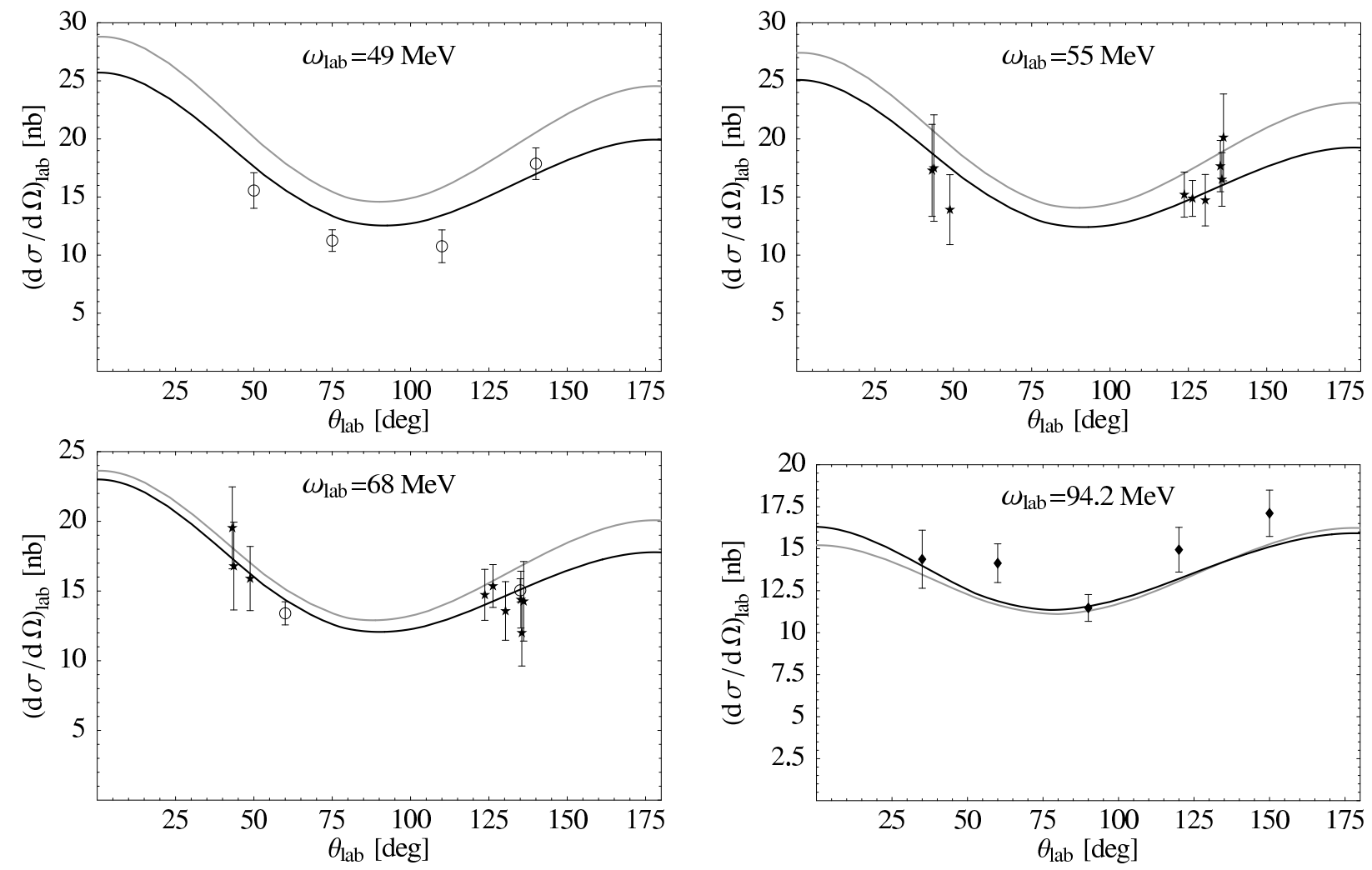

Figure 10: Comparison of our predictions for deuteron Compton scattering (black) with those from Ref. 22] (grey). In both calculations the numbers from the 2-parameter SSE fit to proton data of Ref. 22] are used for the isoscalar polarizabilities. The data are from [7] (circle), 8] (star) and [9] (diamond).

results are parameter-free, as we use the values obtained from proton Compton scattering in [2] for the two a priori unknown parameters $g_{117}$ and $g_{118}$, cf. Fig. [4(f). Obviously we have reached our final goal: a (chirally) consistent calculation of elastic deuteron Compton scattering which describes all existing data reasonably well and also satisfies the lowenergy theorem exactly, cf. Section 2.2.4. If not stated differently, all curves throughout this work have been derived using the NNLO chiral wave function from Ref. [53] with cutoff $\Lambda=650 \mathrm{MeV}$. However, as we demonstrate in Section 3.2, we achieve very similar results with other state-of-the-art wave functions. The values we use for the various input parameters are given in Table 1. The numbers for the short-distance couplings $g_{117}, g_{118}$ are taken from the Baldin-constrained fit [2] of $\alpha_{E}$ and $\beta_{M}$ to the proton Compton data, cf. Eq. (1.2). We use the resulting proton polarizabilities for the neutron analogues as well, as there are no isovector contributions up to third order in the SSE scheme.

There are still minor deviations from the experiments, e.g. our calculation lies slightly above the three $49 \mathrm{MeV}$ data from [7] which have been measured at angles below $120^{\circ}$. However, this is a feature that our calculation has in common with other approaches which also reach the correct static limit, e.g. [20,25,26, 44,46]. At higher energies, the two calculations 


\begin{tabular}{|c|c|c|}
\hline Parameter & Value & Comment \\
\hline$m_{\pi}$ & $139.6 \mathrm{MeV}$ & charged pion mass \\
$m_{N}$ & $938.9 \mathrm{MeV}$ & isoscalar nucleon mass \\
$f^{2}$ & 0.075 & pion-nucleon coupling constant \\
$f_{\pi}$ & $92.4 \mathrm{MeV}$ & pion-decay constant \\
$g_{A}$ & 1.267 & axial coupling constant \\
$\alpha$ & $1 / 137$ & QED fine-structure constant \\
$\mu_{p}$ & 2.795 & magnetic moment of the proton \\
$\mu_{n}$ & -1.913 & magnetic moment of the neutron \\
\hline$\Delta_{0}$ & $271.1 \mathrm{MeV}$ & $N \Delta$ mass splitting \\
$g_{\pi N \Delta}$ & 1.125 & $\pi N \Delta$ coupling constant \\
$b_{1}$ & 4.67 & $\gamma N \Delta$ coupling constant \\
$g_{117}$ & 18.82 & short-distance coupling constant \\
$g_{118}$ & -6.05 & short-distance coupling constant \\
\hline$m_{d}$ & $1875.58 \mathrm{MeV}$ & deuteron mass \\
$B$ & $2.2246 \mathrm{MeV}$ & deuteron binding energy \\
\hline
\end{tabular}

Table 1: $\chi$ EFT parameters determined independently of this work. Magnetic moments are given in nuclear magnetons.

of Ref. [22] and of this work approach each other. This is another important cross-check as the power counting of the calculations of Ref. [22], as well as of Refs. [27 [29], was designed for photon energies of $\omega \sim 100 \mathrm{MeV}$. Consequently, both curves in Fig. 10 describe the 94.2 MeV data from [9] equally well - in fact they nearly lie on top of each other.

Apart from the total result, we discuss the strength of several contributions separately. There are however certain amplitudes which are closely related to each other, e.g. the kinetic energy part of the double commutator, Eq. (2.21), cancels half of the proton seagull in the static limit, cf. Section 2.2.4. The sum of the potential energy part of the commutator and the nine two-body contributions from Fig. 5 is zero in the limit of vanishing photon energy. It stays small in the whole energy range considered in this work, as already observed in Refs. [25, 26, 50]. Therefore we do not separate these contributions from each other. Nevertheless, there are a few issues worth investigating in more detail:

1) The prominent role of the amplitudes $\mathcal{M}_{f i}^{\phi \phi 1,2}$, cf. Eqs. (2.18, 2.19), which include an E1-interaction at both vertices.

2) The importance of the amplitudes $\mathcal{M}_{f i}^{\phi \sigma}$ and $\mathcal{M}_{f i}^{\sigma \sigma}$, with $\sigma$ denoting the coupling to the spin current.

3) The strength of the amplitudes with the explicit Kroll-Ruderman current at one vertex, $\mathcal{M}_{f i}^{\mathrm{KR}}$.

In the upper two panels of Fig. 11 - we investigate the two extreme energies of Fig. 10 - these contributions are successively added to the remaining terms: the single-nucleon amplitudes 
from Figs. 3 and 4 , the two-nucleon diagrams from Fig. 5 and the double-commutator amplitude $\mathcal{M}_{f i}^{\phi \phi 4}$, cf. Eq. (2.21). The amplitude $\mathcal{M}_{f i}^{\phi \phi 3}$ (Eq. (2.20)) is a small correction and has been added to the leading amplitudes $\mathcal{M}_{f i}^{\phi \phi 1,2}$.

Obviously, the amplitudes $\mathcal{M}_{f i}^{\phi \phi}$ are the dominant ones in Fig. 11. This observation holds for both energies considered. However, the amplitudes $\mathcal{M}_{f i}^{\sigma \sigma}$ give also important contributions. The same pattern occurs in the calculation of total deuteron-photodisintegration cross sections, cf. Appendix C. The contributions from $\mathcal{M}_{f i}^{\phi \sigma}$ are nearly negligible. The small size of these terms is due to the fact that the two amplitudes which arise from coupling the two non-gradient parts of $\vec{A}$ to the spin current, cf. Eqs. (2.6), 2.25), largely cancel each other. The diagrams with one photon explicitly coupling to the Kroll-Ruderman current are tiny for low energies, but give a sizeable correction at $94.2 \mathrm{MeV}$. This contribution is stronger in our calculation than it appears in [25,26], which one may partly attribute to the fact that we do not neglect the photon energy in the denominator of the pion propagator of the Kroll-Ruderman current, in contradistinction to [25, 26], see Eqs. (B.40, B.41).

We also give an estimate of the strength of contributions from photons with multipolarity $L=2$, cf. Eq. (A.1). In Ref. [25, 26], these next-to-leading terms in the multipole expansion of the photon field are claimed to be small and therefore have been neglected. However, we slightly disagree from this statement, as can be seen in the lower two panels of Fig. 11, where we compare our full results to curves which only include the $L=L^{\prime}=1$-approximation of the dominant amplitudes $\mathcal{M}_{f i}^{\phi \phi 1,2,3}$ and $\mathcal{M}_{f i}^{\sigma \sigma}$. For low energies, these corrections are certainly negligible, but they are of the order of $10 \%$ in the high-energy regime in the forward and backward direction.

Comparing to Ref. [25, 26], we see the main difference to this work in our systematic $\chi \mathrm{EFT}$ description of the single-nucleon structure. In [25,26], the structure of the nucleon is included only via the static polarizabilities $\alpha_{E}$ and $\beta_{M}$, i.e. via the leading terms of a Taylor expansion of the single-nucleon Compton amplitudes. In our work, these amplitudes have been calculated up to third order in the Small Scale Expansion, as explained in detail in Refs. [2,22], and are included with their full energy dependence. Another advantage of our approach is the treatment of the pion propagator in the pion-exchange diagrams of Fig. 5 , We calculate these diagrams using the full pion propagator, whereas the authors of [25, 26] always assume that the photon energy is small compared to the energy of the virtual pion and therefore may be neglected. This, however, is no longer a good approximation as the photon energy approaches the pion mass. A similar difference occurs in the Kroll-Ruderman amplitudes, as discussed above. Finally, we do not agree with the statement of [25, 26] that $L=2$-contributions are negligible for all amplitudes and energies considered, see Fig. 11 ,

We showed in the last two sections that our calculation provides a consistent description of elastic deuteron Compton scattering below $100 \mathrm{MeV}$. It will be interesting to see whether data from a forthcoming experiment at MAXlab at $\omega \sim 120 \mathrm{MeV}$ are closer to the Green's-function hybrid approach or Weinberg's hybrid approach. There, however, corrections due to the kinematically correct position of the pion-production threshold in analogy to Ref. [2], which are not yet included in our calculation, should not be neglected. Therefore, the $120 \mathrm{MeV}$ curve in the direct comparison of our results at various energies in Fig. 12 is 

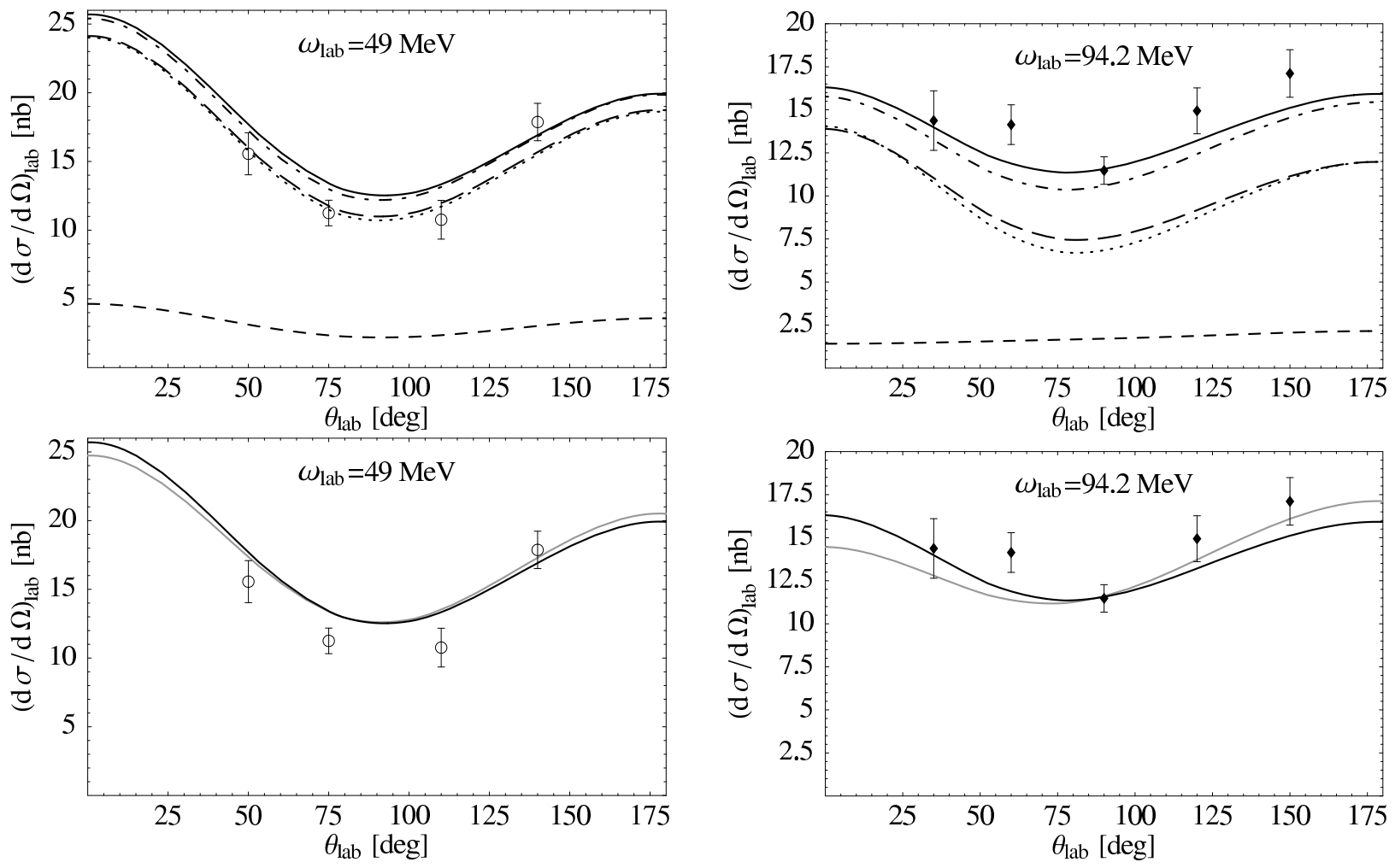

Figure 11: Comparison of separate contributions to our deuteron Compton-scattering results. In the upper panels we compare the full result (solid) to curves with several amplitudes subtracted; the subtracted amplitudes are: $\mathcal{M}_{f i}^{\mathrm{KR}}$ (dotdashed), $\mathcal{M}_{f i}^{\mathrm{KR}}+\mathcal{M}_{f i}^{\sigma \sigma}$ (dotted), $\mathcal{M}_{f i}^{\mathrm{KR}}+\mathcal{M}_{f i}^{\sigma \sigma}+\mathcal{M}_{f i}^{\phi \sigma}$ (longdashed), $\mathcal{M}_{f i}^{\mathrm{KR}}+\mathcal{M}_{f i}^{\sigma \sigma}+\mathcal{M}_{f i}^{\phi \sigma}+\mathcal{M}_{f i}^{\phi \phi 1,2,3}$ (shortdashed). In the lower panels, we compare our full result (black) to a curve where the amplitudes $\mathcal{M}_{f i}^{\sigma \sigma}$ and $\mathcal{M}_{f i}^{\phi \phi}$ have been replaced by their $L=L^{\prime}=1$-approximations (grey). The data are from [7] (circle) and [9] (diamond).

only a qualitative statement about the behavior of the differential cross section for $\omega \rightarrow m_{\pi}$. Hildebrandt et al. 22] estimate this effect to be negligible below $100 \mathrm{MeV}$. We also show our prediction at $\omega_{\text {lab }}=30 \mathrm{MeV}$, which might be considered as a guideline to forthcoming experiments in this energy region [16,17]. It is comparable in magnitude to the $49 \mathrm{MeV}$ curve. Its shape, however, is less asymmetric between the forward and the backward direction, due to the exact forward-backward symmetry of the static cross section, cf. Fig. 9,

In the next two sections, we investigate the sensitivity of our results to the deuteron wave function and the $N N$-potential used. 


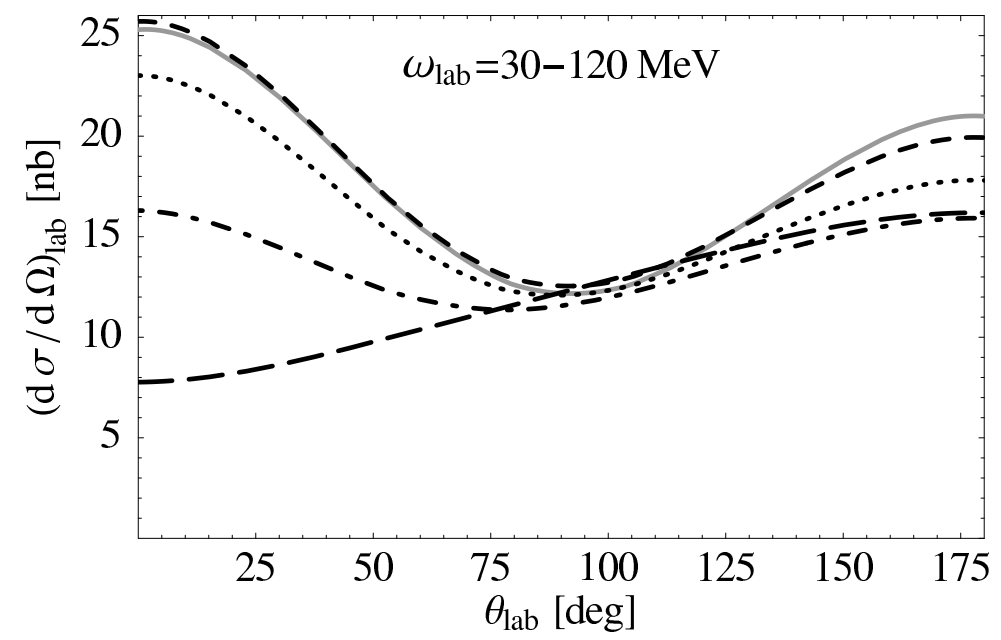

Figure 12: Comparison of our results for differential Compton cross sections at various energies: $30 \mathrm{MeV}$ (grey), $49 \mathrm{MeV}$ (shortdashed), $68 \mathrm{MeV}$ (dotted), $94.2 \mathrm{MeV}$ (dotdashed), $120 \mathrm{MeV}$ (longdashed).

\subsection{Dependence on the Deuteron Wave Function}

As demonstrated in Section 2.2.4, our calculation fulfills the low-energy theorem, Eq. (2.2), which in turn is independent of the deuteron wave function. Therefore and because of the nearly energy-independent offset between the cross sections calculated with the chiral [53] and the AV18-wave function [34, observed in Refs. [22, 40], it is not surprising that the wave-function dependence of our present calculation is also at non-zero energies considerably reduced with respect to Refs. [22, 28, 29]. In fact, the remaining dependence is of the order of $1 \%$ and therefore nearly invisible, cf. Fig. 13, where we compare our cross sections with the two wave functions that turned out as the extreme ones in Ref. [22]: the AV18 [34] and the NNLO $\chi \mathrm{PT}$ [53] wave function (the same observation holds for other state-of-theart deuteron wave functions). This is another important success of our present approach to deuteron Compton scattering, as it demonstrates that our calculation is not sensitive to details of high-energy physics, i.e. short-distance contributions of the wave function, whereas the 10\%-effect observed in Refs. [22,28, 29] manifests a much stronger dependence on shortdistance dynamics than would be expected from a low-energy Effective Field Theory.

\subsection{Dependence on the Potential}

In this section, we investigate briefly the sensitivity to the $n p$-potential used for the rescattering part. Usually, we use the AV18-potential [34] which provides an excellent theoretical description of the Nijmegen partial-wave analysis. We compare here our results achieved with this modern 'high-precision' potential to those of the leading-order chiral potential, which includes only the one-pion exchange and a simple parameterization of short-distance effects via two point-like, momentum-independent contact operators. This potential is given 

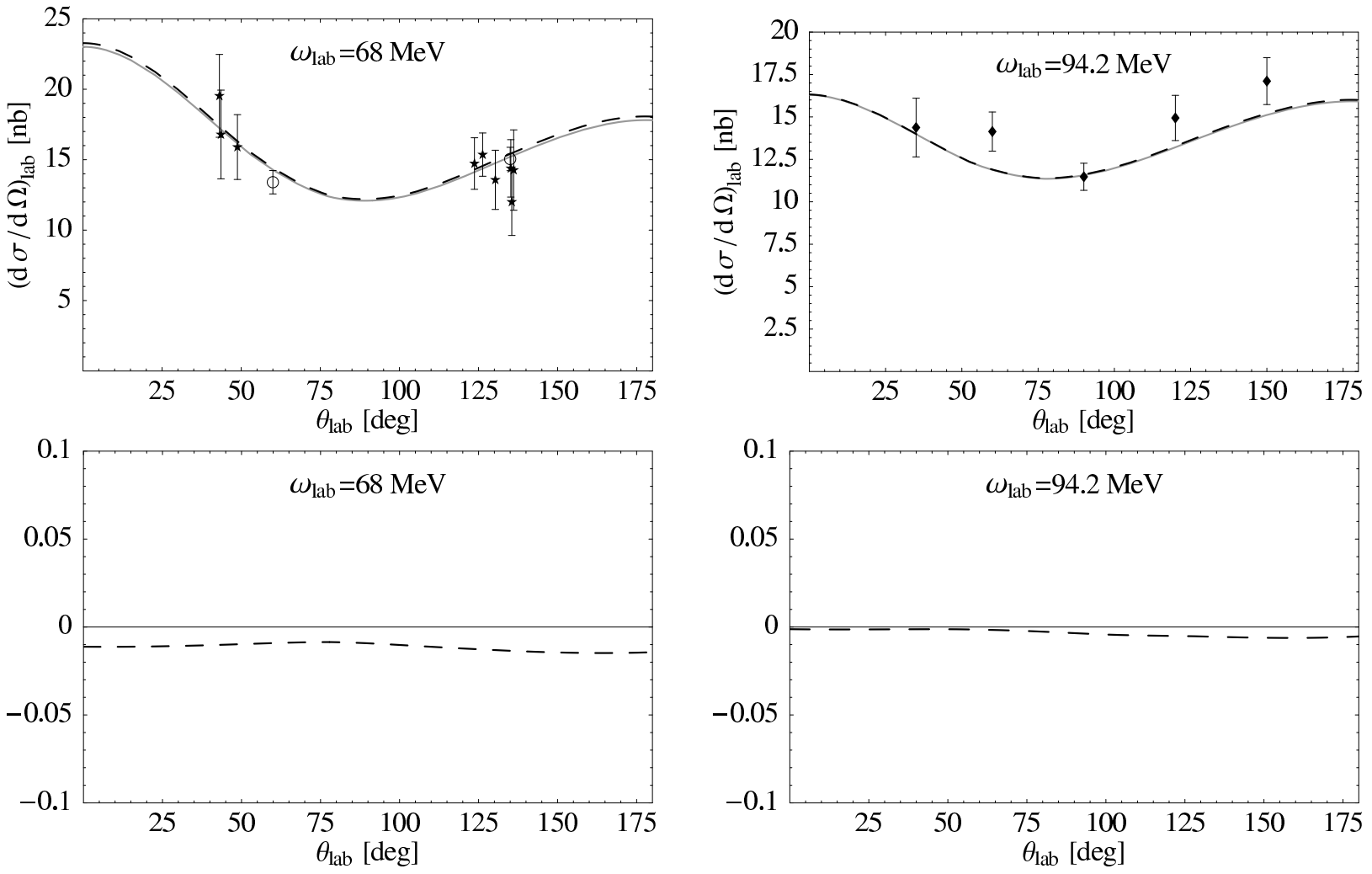

Figure 13: Comparison of our deuteron Compton cross-section results for 68 and $94.2 \mathrm{MeV}$, using two different wave functions: NNLO $\chi \mathrm{PT}$ (grey) [53] and AV18 (dashed) [34]. In the lower two panels we show $\left(\frac{d \sigma}{d \Omega}\right)_{\mathrm{NNLO}} /\left(\frac{d \sigma}{d \Omega}\right)_{\mathrm{AV} 18}-1$.

e.g. in Ref. [57], using a Gaussian regulator in order to render the pion-exchange potential finite at the origin.

At leading order, there are two free parameters, $C_{0}^{1} S_{0}$ and $C_{0}^{d}$, with $d$ denoting the (deuteron) ${ }^{3} S_{1}{ }^{3} D_{1}$ channel. $C_{0}^{1} S_{0}$ has been fixed in [57] via the ${ }^{1} S_{0}$ scattering length, $a_{0} \approx$ $-23.75 \mathrm{fm}, C_{0}^{d}$ to the deuteron binding energy, parameterizing any short-distance physics in the spin-singlet and -triplet channel, respectively. $C_{0}^{1} S_{0}$ and $C_{0}^{d}$, as given in Ref. [57] for the cutoff-value $\Lambda=600 \mathrm{MeV}$, are reported in Table 2 .

\begin{tabular}{|l|l|}
\hline$C_{0}^{1} S_{0}$ & $C_{0}^{d}$ \\
\hline$-0.422 \mathrm{fm}$ & $0.795 \mathrm{fm}$ \\
\hline
\end{tabular}

Table 2: Parameters of the LO chiral potential as determined in [57] for $\Lambda=600 \mathrm{MeV}$.

Even with this rather crude approximation of the neutron-proton interaction, we obtain results close to those of the AV18-potential, cf. Fig. 14. Obviously, the one-pion-exchange potential, adequately regulated for $r \rightarrow 0$, together with a reasonable parameterization of 
the hard core gives an approximation of the potential which is well sufficient for the process under consideration. We conclude that we are mainly sensitive to the long-range part of the potential. Nevertheless, there are small deviations (of the order of $\leq 4 \%$ ) visible in Fig. 14, which justify the application of a more sophisticated potential. These deviations increase with increasing photon energy and scattering angle. However, the correct Thomson limit is reached with any combination of wave-function and two-nucleon rescattering potential, as shown by Arenhövel [49] and recalled in Sec. 2.2.4. Therefore, deviations coming from using different wave-functions or potentials must necessarily increase with increasing energy. One can therefore not simply determine if the seed of discrepancies is a difference between LO potential and phase shifts at low energies, and/or if the different curves arise from the wellexplored poor LO-description of the ${ }^{1} \mathrm{~S}_{0},{ }^{1} \mathrm{P}_{1}$ and ${ }^{3} \mathrm{P}_{0,2}$ partial waves at energies $\sim m_{\pi}$ for some cutoffs, see e.g. [38, 39, 57]. The situation is complicated by the fact that rescattering contributions are small at high energies, see Sec. 3.1. The error induced by neglecting them is comparable to that from using the cruder LO potential in Fig. 14 but has a different angular dependence.
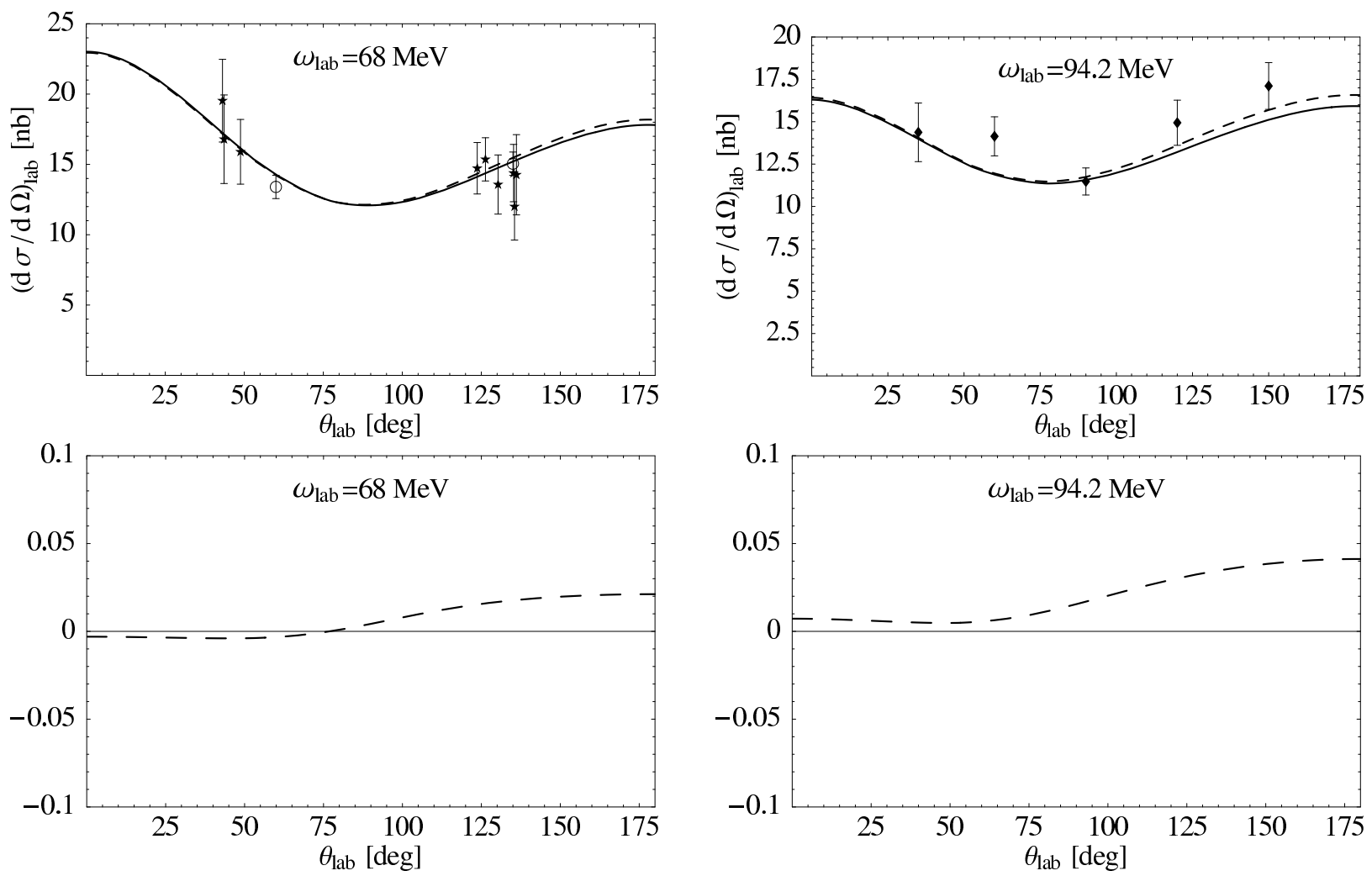

Figure 14: Upper panels: Comparison of our result at $68 \mathrm{MeV}$ and $94.2 \mathrm{MeV}$ using two different $n p$-potentials: the AV18-potential [34] (solid) and the LO chiral potential [57] (dashed). For both curves the chiral wave function [53] has been used. Lower panels: Corresponding error plots, calculated in analogy to Fig. 9. 
Having proven that our calculation is rather insensitive to the choice of the deuteron wave function and the $n p$-potential, we turn now to fits of the isoscalar nucleon polarizabilities (or equivalently the short-distance contributions $g_{117}$ and $g_{118}$ ) to all existing elastic $\gamma d$ data.

\section{Fits of the Isoscalar Polarizabilities}

We saw in Section 3.1 that our results for the elastic deuteron Compton cross sections give a good description of all existing data. Furthermore, the cross-check described in Appendix C, i.e. extracting the total deuteron-photodisintegration cross section from our Compton amplitude, together with the exact reproduction of the low-energy theorem, cf. Section 2.2.4, gives a strong hint that the numerically most important amplitudes have been calculated correctly. Therefore, we now use our deuteron Compton cross sections to fit the static isoscalar nucleon polarizabilities $\alpha_{E}^{s}$ and $\beta_{M}^{s}$ to elastic $\gamma d$ experiments. This corresponds to fitting the coupling constants $g_{117}$ and $g_{118}$ of the two short-distance operators, Fig. 4(f), to elastic $\gamma d$ rather than $\gamma p$ data. We can use all data for the fits, whereas the authors of Ref. [22] had to restrict themselves to the experiments performed around 68 and $94.2 \mathrm{MeV}$ and those of Ref. [28,29] excluded the two $94.2 \mathrm{MeV}$ data in the backward direction in certain fits.

We do a least- $\chi^{2}$ fit, using the chiral NNLO wave function with $\Lambda=650 \mathrm{MeV}$ [53]. Our results for the isoscalar polarizabilities from the global fit to all data read

$$
\begin{aligned}
& \alpha_{E}^{s}=(11.5 \pm 1.4(\text { stat })) \cdot 10^{-4} \mathrm{fm}^{3}, \\
& \beta_{M}^{s}=(3.4 \pm 1.6(\text { stat })) \cdot 10^{-4} \mathrm{fm}^{3} .
\end{aligned}
$$

For now, we only give the statistical error and postpone a discussion of sources and sizes of theoretical errors to the Conclusions. The corresponding $\chi^{2}$ per degree of freedom is

$$
\frac{\chi^{2}}{\text { d.o.f. }}=0.98
$$

with 27 degrees of freedom (4 data points from [7] at $49 \mathrm{MeV}, 9$ from [8] at $55 \mathrm{MeV}, 2$ from [7], 9 from [8] around $68 \mathrm{MeV}$ and 5 from [9] at $94.2 \mathrm{MeV}$, along with two fit parameters). In Fig. 15, we give a contour plot of the achieved $\chi^{2}$, together with the $70 \%$-confidence ellipse.

The plots corresponding to Eq. (4.1), together with the (statistical) error bands, are shown in Fig. 16. The predictions within our Green's-function hybrid approach, using the results for $\alpha_{E}^{p}$ and $\beta_{M}^{p}$ from Eq. (1.2) for the proton and the neutron polarizabilities, describe the data already well, see Fig. 10, It is therefore no surprise that also the fitted curves are in good agreement with experiment. We compare our fit results to "fit IV" from Ref. [28, 29], which is the $\mathcal{O}\left(q^{4}\right) \mathrm{HB} \chi \mathrm{PT}$ fit to all data with central values $\alpha_{E}^{s}=11.5 \cdot 10^{-4} \mathrm{fm}^{3}, \beta_{M}^{s}=$ $0.3 \cdot 10^{-4} \mathrm{fm}^{3}$. As explained in detail in [22, the only sizeable deviations are observed at $94.2 \mathrm{MeV}$ in the backward direction, due to the $\Delta$-resonance diagram, Fig. 4(a), which is not included in the calculation of Ref. [28,29]. In Fig. 17 and Table 3, we also compare to the 2-parameter fit from Ref. [22], which was performed with the $\gamma d \rightarrow \gamma d$ kernel according 

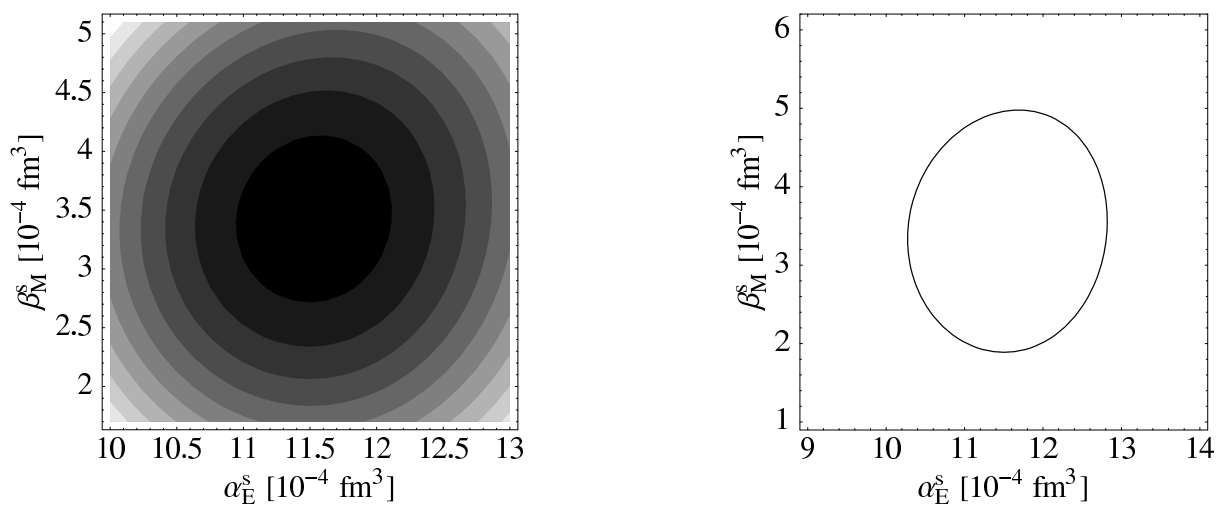

Figure 15: Contour plot of the $\chi^{2}$ achieved in our global 2-parameter fit for $\alpha_{E}^{s}$ and $\beta_{M}^{s}$ (left panel) and the 70\%-confidence ellipse (right panel). The shadings in the left panel are separated by steps of $\Delta \chi^{2}=0.67$.

to third-order SSE, using the chiral wave function [53]. These curves correspond to the fits performed with the "effective" data set, cf. Table 3 and Fig. 11 in [22]. Here we observe a constant offset in the differential cross section at $68 \mathrm{MeV}$, whereas at $94.2 \mathrm{MeV}$ the two curves are quite close to each other, similarly to Fig. 10.

The value of our global fit for $\alpha_{E}^{s}$ is slightly smaller, the one for $\beta_{M}^{s}$ slightly larger than the fit results of Ref. 22], given in Eq. (1.4) and in Table 3, respectively. Nevertheless, both extractions agree well with each other within their error bars, and there is also very good agreement of Eq. (4.1) with the values given in [3], see Eqs. (1.1) and (1.3). Furthermore, we find that the numbers given in Eq. (4.1) add up nearly exactly to the isoscalar Baldin sum rule (see 22] for the proton and neutron sums used as input),

$$
\alpha_{E}^{s}+\left.\beta_{M}^{s}\right|_{\text {world av. }}=(14.5 \pm 0.6) \cdot 10^{-4} \mathrm{fm}^{3},
$$

which has been a serious problem in former extractions [20, 28, 29]. Therefore, in order to reduce the statistical error, we repeat our global fit, using the central sum-rule value as an additional fit constraint. The results are

$$
\begin{aligned}
& \left.\alpha_{E}^{s}\right|_{\text {Baldin }}=(11.3 \pm 0.7(\text { stat }) \pm 0.6(\text { Baldin })) \cdot 10^{-4} \mathrm{fm}^{3}, \\
& \left.\beta_{M}^{s}\right|_{\text {Baldin }}=(3.2 \mp 0.7(\text { stat }) \pm 0.6(\text { Baldin })) \cdot 10^{-4} \mathrm{fm}^{3}
\end{aligned}
$$

with $\chi^{2} /$ d.o.f. $=0.95$. Of course, the central values of Eq. (4.4) are very similar to the ones of Eq. (4.1), due to the nearly perfect agreement of the 2-parameter-fit result with the sum-rule value. However, the statistical error of the Baldin-constrained fit is reduced by about $50 \%$. The plots arising from the global, Baldin-constrained fit, together with the corresponding error bars, are shown in Fig. 18. The central curves are nearly indistinguishable from the ones of Fig. 16. In order to simplify comparison, we sum up our fit results, together with those from Refs. [22, 28, 29] in Table 3. Obviously, all three extractions of the electric 

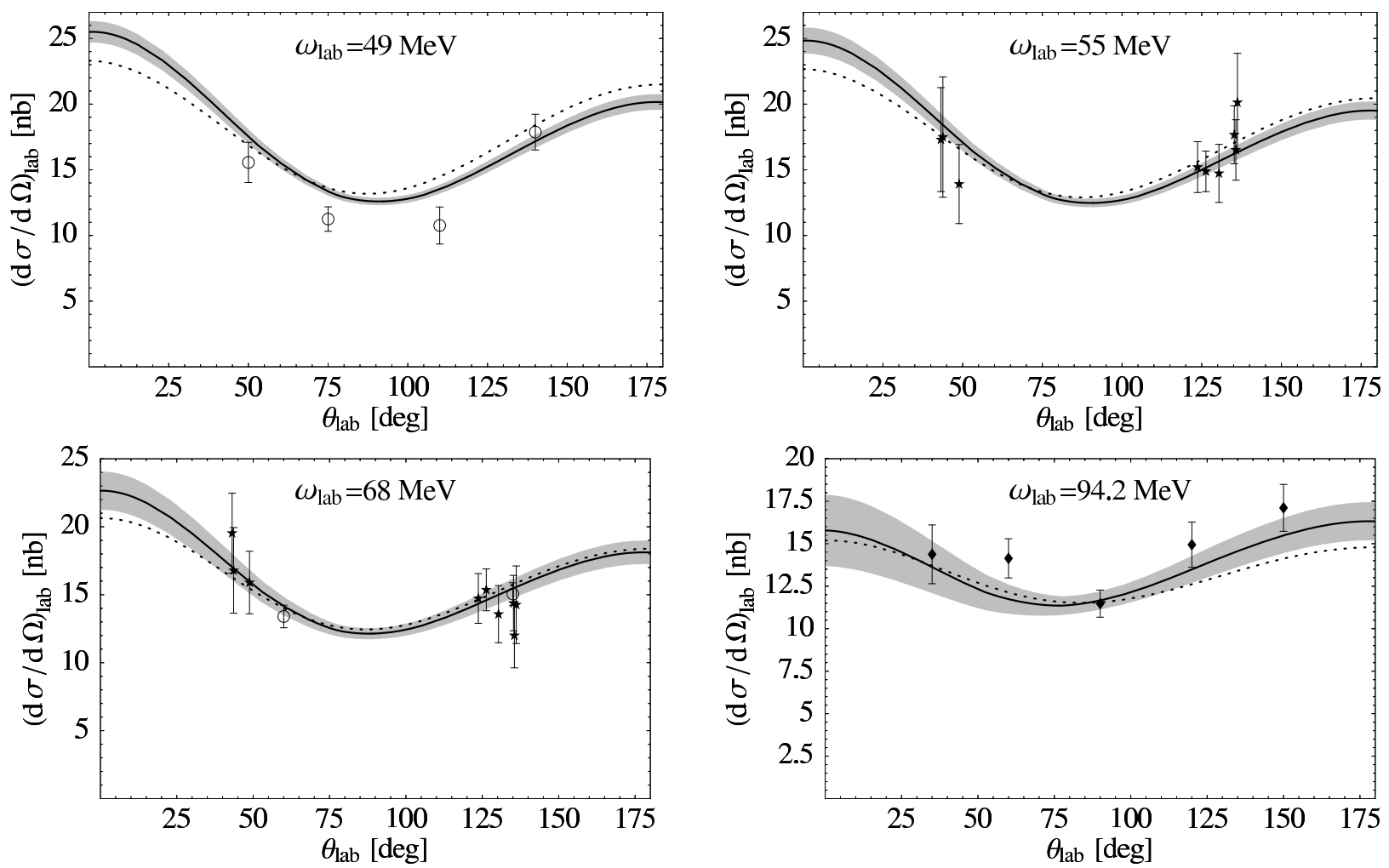

Figure 16: Results from a global fit of $\alpha_{E}^{s}$ and $\beta_{M}^{s}$ to all existing elastic $\gamma d$ data (solid). The grey bands are derived from our statistical errors. The dotted line represents "fit IV", one of the $\mathcal{O}\left(q^{4}\right)$-HB $\chi \mathrm{PT}$ fits from Ref. [28, 29], with central values $\alpha_{E}^{s}=11.5 \cdot 10^{-4} \mathrm{fm}^{3}, \beta_{M}^{s}=$ $0.3 \cdot 10^{-4} \mathrm{fm}^{3}$. For the $\mathcal{O}\left(q^{4}\right)$ calculation the NLO chiral wave function of Ref. [58] has been used, whereas our results were derived with the NNLO-version of this wave function [53].

polarizability agree with each other, whereas determining $\beta_{M}$ without the explicit inclusion of $\Delta$ resonance degrees of freedom yields a negative central value [28, 29], in contradiction to [3,22] and the present work, which show also good agreement in this quantity.

Combining Eqs. (4.1) or (4.4), respectively, with the (Baldin-constrained) fit results of Eq. (1.2), which we extracted from proton data using the SSE framework, we calculate the values for the neutron polarizabilities as

$$
\begin{aligned}
& \alpha_{E}^{n}=(12.0 \pm 2.0(\text { stat }) \pm 0.4(\text { Baldin })) \cdot 10^{-4} \mathrm{fm}^{3}, \\
& \beta_{M}^{n}=(4.0 \pm 2.1(\text { stat }) \pm 0.4(\text { Baldin })) \cdot 10^{-4} \mathrm{fm}^{3}
\end{aligned}
$$

for the 2-parameter fit and

$$
\begin{aligned}
& \left.\alpha_{E}^{n}\right|_{\text {Baldin }}=(11.6 \pm 1.5(\text { stat }) \pm 0.6(\text { Baldin })) \cdot 10^{-4} \mathrm{fm}^{3}, \\
& \left.\beta_{M}^{n}\right|_{\text {Baldin }}=(3.6 \mp 1.5(\text { stat }) \pm 0.6(\text { Baldin })) \cdot 10^{-4} \mathrm{fm}^{3}
\end{aligned}
$$



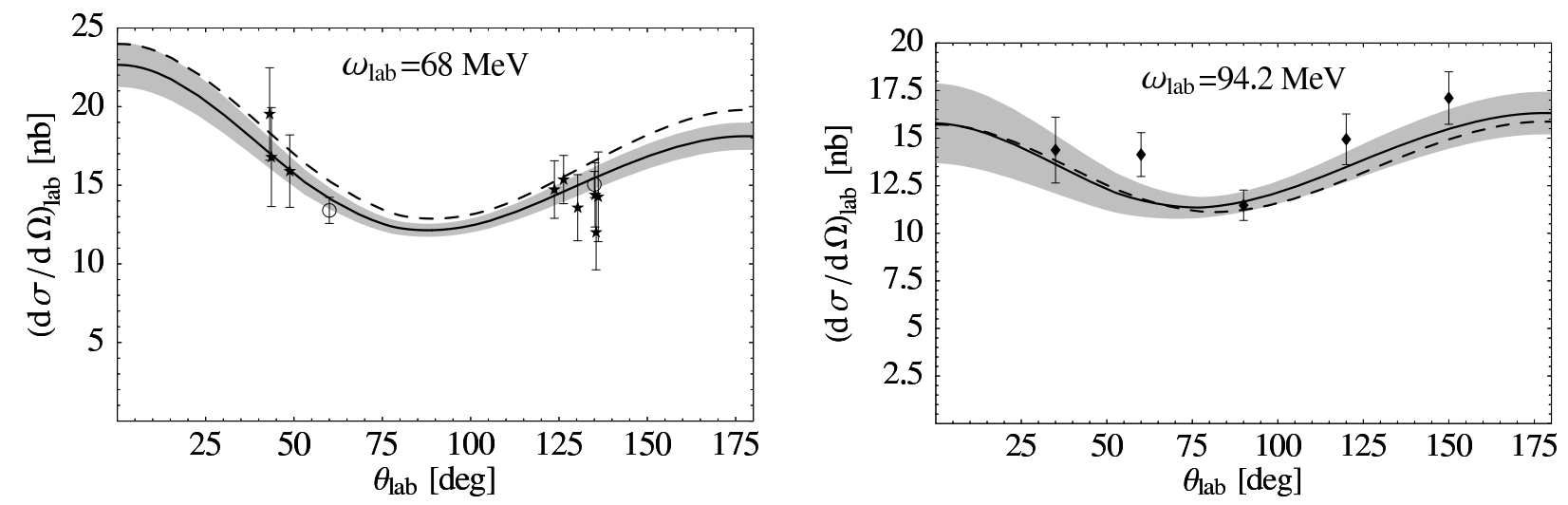

Figure 17: Results from a global fit of $\alpha_{E}^{s}$ and $\beta_{M}^{s}$ to all existing elastic $\gamma d$ data (solid), obtained with the NNLO chiral wave function [53]. The grey bands are derived from our statistical errors. We compare here our results to the 2-parameter-fit results from [22], using the same chiral wave function (dashed).

\begin{tabular}{|c||l|l||c|c|}
\hline \multicolumn{1}{|c||}{} & \multicolumn{2}{c||}{$\alpha_{E}^{s}\left[10^{-4} \mathrm{fm}^{3}\right]$} & \multicolumn{2}{c|}{$\beta_{M}^{s}\left[10^{-4} \mathrm{fm}^{3}\right]$} \\
\hline & 2-parameter fit & 1-parameter fit & 2-parameter fit & 1-parameter fit \\
\hline \hline Ref. [28,29] & $13.0 \pm 1.9_{-1.5}^{+3.9}$ & & $-1.8 \pm 1.9_{-0.9}^{+2.1}$ & \\
\hline Ref. [22] & $12.8 \pm 1.4 \pm 1.1$ & $12.6 \pm 0.8 \pm 0.7$ & $2.1 \pm 1.7 \pm 0.1$ & $1.9 \mp 0.8 \mp 0.7$ \\
\hline \hline this work & $11.5 \pm 1.4$ & $11.3 \pm 0.7$ & $3.4 \pm 1.6$ & $3.2 \mp 0.7$ \\
\hline
\end{tabular}

Table 3: Comparison of the fit results for the isoscalar nucleon polarizabilities $\alpha_{E}^{s}$ and $\beta_{M}^{s}$, achieved within $\mathcal{O}\left(\epsilon^{3}\right)$ SSE [22] (Weinberg hybrid approach), $\mathcal{O}\left(p^{4}\right) \mathrm{HB} \chi \mathrm{PT}$ [28, 29] (Weinberg hybrid approach) and the Green's-function hybrid approach presented in this work, respectively. The first error-bar is statistical; a second error bar denotes the systematic uncertainty from the wave-function dependence [22,28, 29] and the arbitrariness as to which data are included in the fit [28, 29]. In the one-parameter fits, the error induced by the Baldin sum rule is not shown.

for the fit of the isoscalar polarizabilities including the Baldin constraint. We consider these values to be as reliable as those from the quasi-free Compton experiment of Ref. [21], as the isoscalar polarizabilities, from which they are derived, have been determined by fitting our deuteron Compton calculation, which fulfills the low-energy theorem, to all existing elastic deuteron Compton-scattering data. That means there is no restriction on either the energy, as in Ref. [22], or on the angle, like in Ref. [28, 29]. From these values we deduce that the magnetic response of the neutron is comparable to that of the proton and that nucleons are paramagnetic. We also conclude that the isovector polarizabilities are considerably smaller than the isoscalar ones. In other words, our analysis shows that within the precision of the currently existing data, elastic Compton scattering from the proton and the deuteron is in 

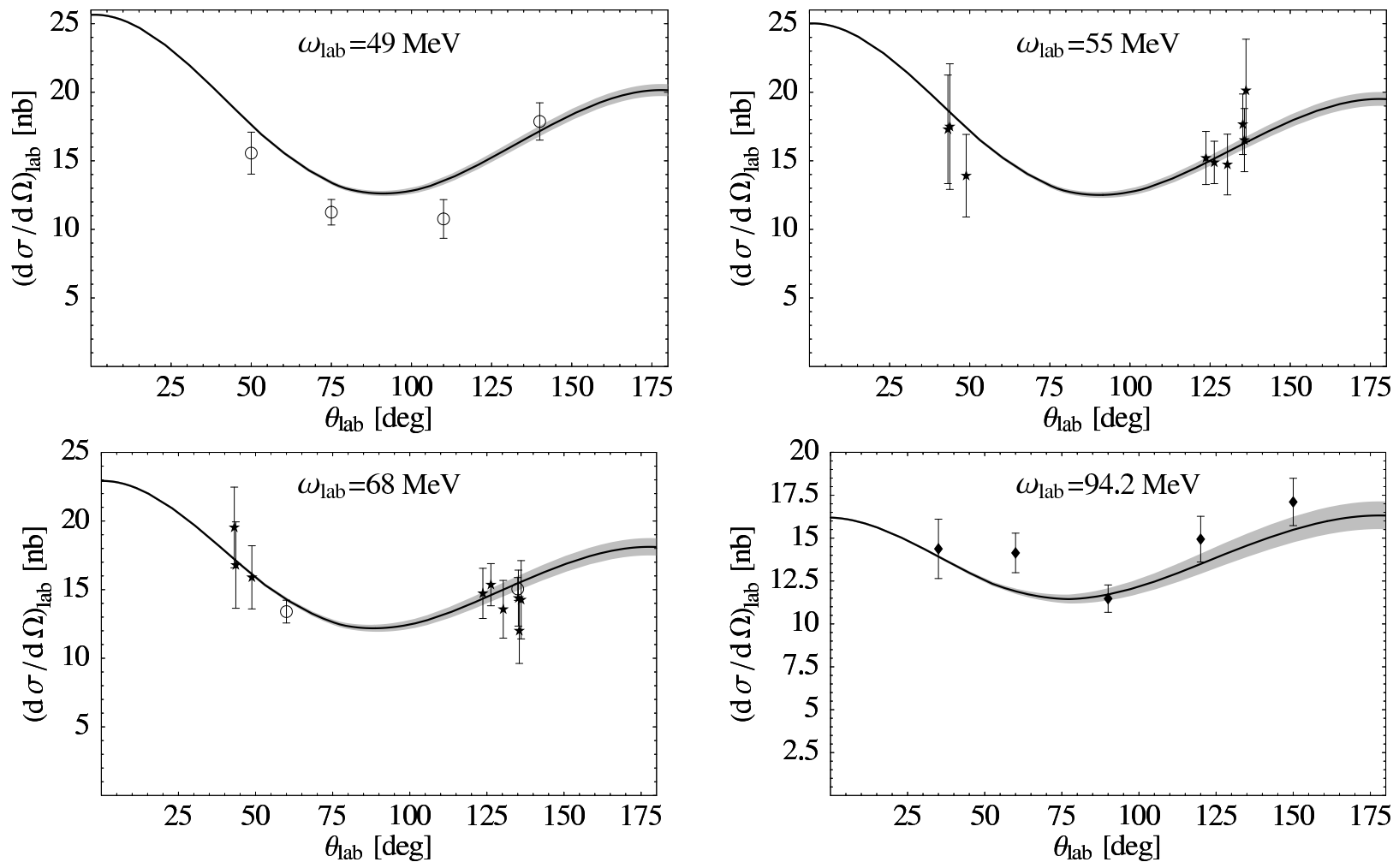

Figure 18: Results from a global fit of $\alpha_{E}^{s}$ to all existing elastic $\gamma d$ data, using the chiral wave function [53]. $\beta_{M}^{s}$ is fixed via the Baldin sum rule, Eq. (4.3). The grey bands are derived from our statistical errors.

agreement with

$$
\alpha_{E}^{p} \approx \alpha_{E}^{n}, \quad \beta_{M}^{p} \approx \beta_{M}^{n}
$$

These findings agree with those of Refs. [3, 22].

\section{Conclusion}

In this work we examined elastic Compton scattering from the deuteron. Diagrams without an intermediate two-nucleon state have been calculated up to next-to-leading order in the Small Scale Expansion, an Effective Field Theory with nucleons, pions and the $\Delta(1232)$ resonance as explicit degrees of freedom. Those diagrams including the propagation of the two-nucleon system between the two photon vertices have been calculated using Green'sfunction methods. Therefore, and because of the fact that we use deuteron wave functions that have been derived from state-of-the-art $N N$-potentials, we refer to this approach as the "Green's-function hybrid approach". For the photon coupling we make use of Siegert's theorem [48, which is well-known to guarantee the exact static limit, see e.g. [25, 26, 49, 50]. 
In Section 3.1, we show that we have achieved a consistent description of elastic deuteron Compton scattering which is valid from $0 \mathrm{MeV}$ up to $\omega \sim 100 \mathrm{MeV}$. The advantage of our calculation with respect to Refs. [22,27, 29] is that we obtain a good description of the data published below $60 \mathrm{MeV}$. This improvement is of course connected to the correct static limit, which had not been obtained in those publications. Therefore, other calculations reaching this limit and thus being manifestly gauge invariant are also able to describe the low-energy data well, see e.g. [20, 25, 26, 44, 46]. However, we achieve good agreement also with high-energy data, measured around $94.2 \mathrm{MeV}$ [9]. In this energy regime the work of Ref. [25, 26] fails and the "pion-less" Effective Field Theory used in [44 46] is inapplicable. The authors of [20] have also problems to describe the data in the backward direction without introducing surprisingly large isovector polarizabilities. It was already shown in [22] that the main difference between their approach and ours is in the energy dependence of the resonant Compton multipoles, which is well captured in our calculation due to the inclusion of explicit $\Delta$-resonance degrees of freedom, whereas the authors of Ref. 20] include the polarizabilities only via the leading and subleading terms of a Taylor expansion in the photon energy.

There are a number of ways to check the theoretical uncertainties of our calculation. As demonstrated in Section 3.2 and Fig. 13, the error induced by dependence on the deuteron wave function is tiny and may well be set to zero, while it is sizeable in the extractions of Refs. [22, 28, 29]. In Section 3.3 and Fig. 14, we compared the cross-sections derived using either the AV18-potential, or the much cruder one-pion exchange potential, regulated to reproduce the deuteron binding energy and ${ }^{1} S_{0}$-scattering length. This may for the time being serve as LO potential of $\chi \mathrm{EFT}$. We find that the magnetic polarizability using this potential is less than $1 \cdot 10^{-4} \mathrm{fm}^{3}$ larger than using AV18, and the electric polarizability decreases by practically the same amount. As it is safe to assume that AV18 is a better representative of a fully self-consistent $\chi \mathrm{EFT}$ potential at the level of our calculation (next-to-leading order) than one-pion exchange, we classify uncertainties induced by the potential as negligible. A third way to estimate the errors from the theory side is to compare to our previous calculation [22]. With the single-nucleon sector treated identically to the approach presented here, its only difference is that the interaction kernel is expanded perturbatively. As argued in Section 3.1, this approximation is justified in the régime $\omega \sim m_{\pi}$. There, both results should thus coincide up to higher-order corrections stemming from the different treatment of the two-nucleon sector. Figure 17 and in particular the extracted polarizabilities in Table 3 show that both approaches agree well within the statistical error, and do not deviate by more than $1.3 \cdot 10^{-4} \mathrm{fm}^{3}$. Finally, higher-order corrections to the energy-dependence of the polarizabilities themselves were in SSE estimated by naïve dimensional analysis to be less than $1 \cdot 10^{-4} \mathrm{fm}^{3}[22]$. All these interdependent estimates suggest a theory uncertainty of our extraction of $\pm 1 \cdot 10^{-4} \mathrm{fm}^{3}$ as appropriate. This is comparable with the statistical error.

Having achieved a good description of all elastic deuteron Compton-scattering data enabled us therefore to perform a global fit of the isoscalar polarizabilities to all existing data points, published in [7 9]. Our 2-parameter-fit results are (see Table 3])

$$
\begin{aligned}
& \alpha_{E}^{s}=(11.5 \pm 1.4(\text { stat }) \pm 1(\text { theory })) \cdot 10^{-4} \mathrm{fm}^{3}, \\
& \beta_{M}^{s}=(3.4 \pm 1.6(\text { stat }) \pm 1(\text { theory })) \cdot 10^{-4} \mathrm{fm}^{3} .
\end{aligned}
$$


We note that the numbers of Eq. (5.1) are very close to the fit results (1.2) for the proton polarizabilities, determined in [2] within the SSE framework. This leaves little space for large isovector polarizabilities. Furthermore they agree extraordinarily well with the isoscalar Baldin-sum-rule value, Eq. (4.3). Therefore, in order to reduce the statistical error, we repeated our fits including this constraint:

$$
\begin{aligned}
& \left.\alpha_{E}^{s}\right|_{\text {Baldin }}=(11.3 \pm 0.7(\text { stat }) \pm 0.6(\text { Baldin }) \pm 1(\text { theory })) \cdot 10^{-4} \mathrm{fm}^{3}, \\
& \left.\beta_{M}^{s}\right|_{\text {Baldin }}=(3.2 \mp 0.7(\text { stat }) \pm 0.6(\text { Baldin }) \pm 1(\text { theory })) \cdot 10^{-4} \mathrm{fm}^{3} .
\end{aligned}
$$

Combining Eqs. (5.1) or (5.2), respectively, with the proton numbers of Eq. (1.2), we obtained the neutron polarizabilities

$$
\begin{aligned}
& \alpha_{E}^{n}=(12.0 \pm 2.0(\text { stat }) \pm 0.4(\text { Baldin }) \pm 1(\text { theory })) \cdot 10^{-4} \mathrm{fm}^{3}, \\
& \beta_{M}^{n}=(4.0 \pm 2.1(\text { stat }) \pm 0.4(\text { Baldin }) \pm 1(\text { theory })) \cdot 10^{-4} \mathrm{fm}^{3}
\end{aligned}
$$

for the 2-parameter fit and

$$
\begin{aligned}
& \left.\alpha_{E}^{n}\right|_{\text {Baldin }}=(11.6 \pm 1.5(\text { stat }) \pm 0.6(\text { Baldin }) \pm 1(\text { theory })) \cdot 10^{-4} \mathrm{fm}^{3}, \\
& \left.\beta_{M}^{n}\right|_{\text {Baldin }}=(3.6 \mp 1.5(\text { stat }) \pm 0.6(\text { Baldin }) \pm 1(\text { theory })) \cdot 10^{-4} \mathrm{fm}^{3}
\end{aligned}
$$

for the 1-parameter fit including the Baldin constraint. In both fits the fact that our deuteron Compton-scattering calculation is applicable in the whole energy range from $0 \mathrm{MeV}$ to $100 \mathrm{MeV}$ enables us to include all data into our fit of the isoscalar polarizabilities. From these results we deduce that the neutron is paramagnetic and that proton and neutron behave rather similarly when exposed to external electromagnetic fields. In both points our deuteron Compton calculation agrees with Refs. [3, 21, 22] and with the Chiral Perturbation Theory prediction that contributions to the isovector polarizabilities only start beyond leading-oneloop order. This also justifies again that the two iso-scalar short-distance contributions to the electric and magnetic scalar polarizabilities in Sec. 2.1 are the smallest set which needs to be promoted by one order as in Refs. 2, 22] to describe the data, given present uncertainties.

Our results for $\alpha_{E}$ and $\beta_{M}$ are in excellent agreement with those from a comprehensive, experimental analysis of all quasi-free data [3]. That analysis did, however, not include elastic deuteron Compton scattering due to the alleged discrepancy between theory and elastic $\gamma d$ experiments. This discrepancy has now been resolved, both in [22] and in the Green's-function hybrid approach presented in this work.

Finally, we strongly advocate enlarging the data base for elastic Compton scattering on the deuteron. If further experiments, as planned or running at TUNL/HI $\gamma \mathrm{S}$ [10, 15, at the S-DALINAC [18] and at MAXlab [16, 17, provide additional quality data below the pion threshold, the increased statistics would reduce the statistical error in our fit of the isoscalar polarizabilities. Recall that the statistical errors of the two-parameter fit (5.1) are larger than our theoretical uncertainty, and that the combined statistical and Baldin-sumrule error of the one-parameter fit (5.2) is comparable to it. These errors are increased 
by the systematic experimental uncertainties which may be estimated more comfortably if more experiments using different methods were available. On the theory side, a wider effort is under way to describe elastic Compton scattering on the proton, deuteron and ${ }^{3}$ He from the Thomson limit to well into the $\Delta$-resonance region in one model-independent, unified framework. We focus at present on improving the accuracy by a full next-to-nextto-leading order calculation with nucleons, pions and the $\Delta(1232)$ as dynamical, effective degrees of freedom [59,60]. Clearly, reduced error bars in coherent Compton scattering from the deuteron would enable us to quantify by how much proton and neutron polarizabilities differ.

\section{Acknowledgments}

The authors acknowledge helpful discussions with Wolfram Weise, Norbert Kaiser, Daniel R. Phillips and Andreas Nogga. We are also grateful to G. Feldman, J. Friar, G. Miller and D.-O. Riska for useful comments made on several points. We thank D.R. Phillips for providing us with his deuteron Compton code and E. Epelbaum and V. Stoks for their deuteron wave functions. A number of our colleagues have encouraged us to publish these results even after a substantial temporal hiatus which is entirely our fault. We are grateful to the ECT* for its hospitality and financial support during the workshops "Two-photon physics" (RPH and HWG) and "Nuclear forces and QCD: never the twain shall meet?" (HWG and TRH). HWG also acknowledges support and discussions during the TUNL/HI $\gamma \mathrm{S}$ mini-workshop on Compton scattering. This work has been supported in part by the Bun-

desministerium für Forschung und Technologie, by Deutsche Forschungsgemeinschaft under contract GR1887/2-2, by a National Science Foundation CAREER award PHY-0645498 (HWG) and US-Department of Energy grant DE-FG02-95ER-40907 (HWG). 


\section{A Multipole Expansion of the Photon Field}

For the photon field $\vec{A}(\vec{\xi})$, we use the multipole expansion derived in [25, 26] in analogy to [54, see also Ref. [40]. The result is

$$
\begin{aligned}
\hat{\epsilon}_{\lambda} \mathrm{e}^{i \vec{k} \cdot \vec{\xi}} & =\sum_{L=1}^{\infty} \sum_{M=-L}^{L} d_{M, \lambda}^{L}(\theta) i^{L} \sqrt{\frac{2 \pi(2 L+1)}{L(L+1)}} \\
& \times\left\{-\frac{i}{\omega} \vec{\nabla}_{\xi} \psi_{L}(\omega \xi) Y_{L M}(\hat{\xi})-i \omega \vec{\xi} j_{L}(\omega \xi) Y_{L M}(\hat{\xi})-\lambda \vec{L} Y_{L M}(\hat{\xi}) j_{L}(\omega \xi)\right\} .
\end{aligned}
$$

$\hat{\epsilon}_{\lambda}$ denotes the photon polarization vector in the spherical (helicity) basis. The functions $d_{M, \lambda}^{L}(\theta)$ are the Wigner $D$-functions $D_{M, \lambda}^{L}(\alpha, \theta, \gamma)$ for $\alpha=\gamma=0$, angular momentum $L$ and magnetic quantum number $M ; Y_{L M}$ denote the spherical harmonics and $\psi_{L}(\omega r)=$ $\left(1+r \frac{d}{d r}\right) j_{L}(\omega r)$ with the spherical Bessel functions $j_{L}(z)=\sqrt{\frac{\pi}{2 z}} J_{L+\frac{1}{2}}(z)$ and $J_{L}(z)$ the Bessel functions of the first kind. In the static (long-wavelength) limit only the gradient term in Eq. (A.1) survives, as for $\omega \rightarrow 0, j_{1}(\omega r) \rightarrow \frac{1}{3} \omega r$ and $\psi_{1}(\omega r) \rightarrow \frac{2}{3} \omega r$. This term turns out to be the dominant part of the photon field for all energies under consideration. Therefore, like in Ref. [25, 26], we define two scalar functions

$$
\begin{aligned}
& \phi_{i}(\vec{r})=-\sum_{L=1}^{\infty} \sum_{M=-L}^{L} \delta_{M, \lambda_{i}} \frac{i^{L+1}}{\omega} \sqrt{\frac{2 \pi(2 L+1)}{L(L+1)}} \psi_{L}(\omega r) Y_{L M}(\hat{r}), \\
& \phi_{f}(\vec{r})=\sum_{L^{\prime}=1}^{\infty} \sum_{M^{\prime}=-L^{\prime}}^{L^{\prime}}(-1)^{L^{\prime}-\lambda_{f}} d_{M^{\prime},-\lambda_{f}}^{L^{\prime}}(\theta) \frac{i^{L^{\prime}+1}}{\omega} \sqrt{\frac{2 \pi\left(2 L^{\prime}+1\right)}{L^{\prime}\left(L^{\prime}+1\right)}} \psi_{L^{\prime}}(\omega r) Y_{L^{\prime} M^{\prime}}(\hat{r}),
\end{aligned}
$$

which allow us to write

$$
\begin{aligned}
\hat{\epsilon}_{\lambda_{i}} \mathrm{e}^{i \vec{k}_{i} \cdot \vec{\xi}} & \approx \vec{\nabla} \phi_{i}(\vec{\xi}), \\
\hat{\epsilon}_{\lambda_{f}}^{*} \mathrm{e}^{-i \vec{k}_{f} \cdot \vec{\xi}} & \approx \vec{\nabla} \phi_{f}(\vec{\xi}) .
\end{aligned}
$$

We want to decompose the photon field in its electric and magnetic part. Therefore, we write Eq. (A.1) in Eq. (2.6) schematically as $\vec{A}=\vec{\nabla} \phi+\vec{A}^{(1)}+\vec{A}^{(2)}$ with

$$
\begin{aligned}
\vec{A}^{(1)}(\vec{\xi}) & =-\sum_{\vec{k}, \lambda= \pm 1} \sum_{L=1}^{\infty} \sum_{M=-L}^{L} \lambda \sqrt{\frac{2 \pi(2 L+1)}{L(L+1)}} i^{L} j_{L}(\omega \xi) \vec{L} Y_{L M}(\hat{\xi}) \\
& \times\left[a_{\vec{k}, \lambda} \delta_{M, \lambda}-a_{\vec{k}, \lambda}^{\dagger}(-1)^{L+\lambda} d_{M,-\lambda}^{L}(\theta)\right] \\
\vec{A}^{(2)}(\vec{\xi}) & =-\sum_{\vec{k}, \lambda= \pm 1} \sum_{L=1}^{\infty} \sum_{M=-L}^{L} \sqrt{\frac{2 \pi(2 L+1)}{L(L+1)}} i^{L+1} \omega \vec{\xi} j_{L}(\omega \xi) Y_{L M}(\hat{\xi}) \\
& \times\left[a_{\vec{k}, \lambda} \delta_{M, \lambda}-a_{\vec{k}, \lambda}^{\dagger}(-1)^{L+\lambda} d_{M,-\lambda}^{L}(\theta)\right] .
\end{aligned}
$$


$\vec{A}^{(1)}$ constitutes the magnetic part of the photon field, $\vec{\nabla} \phi+\vec{A}^{(2)}$ is the electric field [54]. The operators $a_{\vec{k}, \lambda}^{\dagger}\left(a_{\vec{k}, \lambda}\right)$ create (destroy) a photon with momentum $\vec{k}$ and polarization $\lambda$.

\section{B Calculation of Diagrams with Intermediate $N N$-Scattering}

Here we give details about the calculation of the diagrams sketched in Fig. 6. Special emphasis is put on the construction of the two-nucleon Green's function, drawing substantially from Ref. [25, 26].

$\mathcal{M}_{f i}^{\phi \phi 1}$ (Eq. (2.18) ) will be calculated first, in analogy to Ref. [25, 26]. Defining the shortcut $E_{0} \equiv \omega+\frac{\omega^{2}}{2 m_{d}}-B$ and neglecting prefactors for the moment we can write this amplitude as

$$
\mathcal{M}_{f i}^{\phi \phi 1} \propto \sum_{C} \frac{\left\langle d_{f}\left|\psi_{L^{\prime}} Y_{L^{\prime} M^{\prime}}\right| C\right\rangle\left\langle C\left|\psi_{L} Y_{L M}\right| d_{i}\right\rangle}{E_{0}-E_{C}},
$$

where we suppressed the sums over $L, M$ and $L^{\prime}, M^{\prime}$ for brevity, cf. Eq. (A.2). Each wave function can be separated into a radial part, denoted by the index 'rad', and an angular part, denoted by a hat. Furthermore, $|C\rangle$ is an eigenstate to $H^{n p}$ with eigenvalue $E_{C}$, so we can write

$$
\mathcal{M}_{f i}^{\phi \phi 1} \propto \sum_{C_{\mathrm{rad}} \hat{C}}\left\langle d_{f \mathrm{rad}} \hat{d}_{f}\left|\psi_{L^{\prime}} Y_{L^{\prime} M^{\prime}} \frac{1}{E_{0}-H_{\hat{C}}^{n p}}\right| C_{\mathrm{rad}} \hat{C}\right\rangle\left\langle C_{\mathrm{rad}} \hat{C}\left|\psi_{L} Y_{L M}\right| d_{i \mathrm{rad}} \hat{d}_{i}\right\rangle
$$

$\hat{C}$ is used as a shorthand notation for all angular quantum numbers of the intermediate state, i.e. $|\hat{C}\rangle=\left|L_{C} S_{C} J_{C} M_{C}\right\rangle$. Separating the radial from the angular part of $|C\rangle$ and inserting two complete sets of radial states $|r\rangle$ and $\left|r^{\prime}\right\rangle$ we get

$$
\begin{aligned}
\mathcal{M}_{f i}^{\phi \phi 1} & \propto \sum_{C_{\mathrm{rad}} \hat{C}} \int_{0}^{\infty} \int_{0}^{\infty} r^{2} d r r^{2} d r^{\prime}\left\langle\hat{d}_{f}\left|Y_{L^{\prime} M^{\prime}}\right| \hat{C}\right\rangle\left\langle d_{f \mathrm{rad}}\left|\psi_{L^{\prime}}\right| r^{\prime}\right\rangle \\
& \times\left\langle r^{\prime}\left|\frac{1}{E_{0}-H_{\hat{C}}^{n p}}\right| C_{\mathrm{rad}}\right\rangle\left\langle C_{\mathrm{rad}} \mid r\right\rangle\left\langle r\left|\psi_{L}\right| d_{i \mathrm{rad}}\right\rangle\left\langle\hat{C}\left|Y_{L M}\right| \hat{d}_{i}\right\rangle .
\end{aligned}
$$

For the deuteron $(J=1)$ wave function, we use the notation $\Psi_{1 m}(\vec{r})=\sum_{l=0,2} \frac{u_{l}(r)}{r} \mathcal{Y}_{m}^{l 11}(\hat{r})$ with $u_{0}(r)\left(u_{2}(r)\right)$ denoting the usual radial wave functions $u(r)(w(r))$ in position space, cf. e.g. Ref. [55]. The indices of the angular wave functions $\mathcal{Y}$ are $l 11$ for orbital angular momentum, spin and total angular momentum of the deuteron state, $m \in\{-1,0,1\}$. Now we can write Eq. (B.3. $)$ as

$$
\begin{aligned}
\mathcal{M}_{f i}^{\phi \phi 1} & \propto \sum_{\hat{C}} \sum_{l=0,2} \sum_{l^{\prime}=0,2}\left\langle l^{\prime} 11 M_{f}\left|Y_{L^{\prime} M^{\prime}}\right| \hat{C}\right\rangle\left\langle\hat{C}\left|Y_{L M}\right| l 11 M_{i}\right\rangle \\
& \times \iint r d r r^{\prime} d r^{\prime} u_{l^{\prime}}\left(r^{\prime}\right) \psi_{L^{\prime}}\left(\frac{\omega r^{\prime}}{2}\right)\left\langle r^{\prime}\left|\frac{1}{E_{0}-H_{\hat{C}}^{n p}}\right| r\right\rangle \psi_{L}\left(\frac{\omega r}{2}\right) u_{l}(r),
\end{aligned}
$$


where we have removed the sum over $C_{\text {rad }}$. Integrals without limits are always integrated from 0 to infinity throughout this work.

We now have to evaluate the double integral in Eq. (B.4), including the Green's function

$$
G_{\hat{C}}\left(r, r^{\prime} ; E_{0}\right)=\left\langle r^{\prime}\left|\frac{1}{E_{0}-H_{\hat{C}}^{n p}}\right| r\right\rangle .
$$

However, we need to evaluate the integral for arbitrary functions of $r$. Therefore we describe how to calculate

$$
\mathcal{I}_{f i}^{l l^{\prime} \hat{C}}=\iint r d r r^{\prime} d r^{\prime} u_{l^{\prime}}\left(r^{\prime}\right) J_{f}\left(r^{\prime}\right) G_{\hat{C}}\left(r, r^{\prime} ; E_{0}\right) J_{i}(r) u_{l}(r)
$$

We do so in two steps and define

$$
\chi_{f}^{l^{\prime} \hat{C}}(r) \equiv \int r^{\prime} d r^{\prime} u_{l^{\prime}}\left(r^{\prime}\right) J_{f}\left(r^{\prime}\right) G_{\hat{C}}\left(r, r^{\prime} ; E_{0}\right)
$$

Once we have solved this first part, it is easy to numerically calculate the remaining integral

$$
\mathcal{I}_{f i}^{l l^{\prime} \hat{C}}=\int r d r u_{l}(r) J_{i}(r) \chi_{f}^{l^{\prime} \hat{C}}(r) .
$$

In order to find the function $\chi_{f}^{l^{\prime} \hat{C}}(r)$ - in the following we use the abbreviation $\chi_{\hat{C}}(r)$ for brevity - we first note that

$$
\left(E_{0}-H_{\hat{C}}^{n p}\right) G_{\hat{C}}\left(r, r^{\prime} ; E_{0}\right)=\left\langle r^{\prime} \mid r\right\rangle=\frac{\delta\left(r^{\prime}-r\right)}{r^{2}}
$$

Eq. (B.9) defines the Green's function corresponding to Schrödinger's equation with a central potential and the Hamiltonian

$$
H_{\hat{C}}^{n p}=\frac{\vec{p}^{2}}{m_{N}}+V_{\hat{C}}(r)=-\frac{1}{m_{N}}\left(\frac{1}{r} \frac{d^{2}}{d r^{2}} r\right)+\frac{L_{C}\left(L_{C}+1\right)}{m_{N} r^{2}}+V_{\hat{C}}(r) .
$$

The dependence of the potential on the quantum numbers of the interim state $|C\rangle$ is shown explicitly. Note that the neutron-proton potential contains a tensor part and therefore not only depends on the distance $r$ but on the vector $\vec{r}$. The tensor force mixes e.g. the deuteron $s$ - and $d$-states in Schrödinger's equation. Nevertheless, on the level of the Green's function this matrix equation decouples, cf. Ref. [55]. The decoupling of Eq. (B.9) guarantees that only the diagonal terms of the tensor force contribute. Therefore, the orbital angular momentum is well defined, which allows us to replace $\vec{L}^{2} \rightarrow L_{C}\left(L_{C}+1\right)$ in Eq. (B.10).

Eqs. (B.9) and (B.10) combine to

$$
\left[E_{0}+\frac{1}{m_{N}} \frac{d^{2}}{d r^{2}}-\frac{L_{C}\left(L_{C}+1\right)}{m_{N} r^{2}}-V_{\hat{C}}(r)\right] r G_{\hat{C}}\left(r, r^{\prime} ; E_{0}\right)=\frac{\delta\left(r^{\prime}-r\right)}{r},
$$


which e.g. in the deuteron case reduces to the two differential equations

$$
\begin{gathered}
{\left[E_{0}+\frac{1}{m_{N}} \frac{d^{2}}{d r^{2}}-V_{\text {cent }}(r)\right] r G_{0}\left(r, r^{\prime} ; E_{0}\right)=\frac{\delta\left(r^{\prime}-r\right)}{r},} \\
{\left[E_{0}+\frac{1}{m_{N}} \frac{d^{2}}{d r^{2}}-\frac{6}{m_{N} r^{2}}-V_{\text {cent }}(r)+2 V_{\text {ten }}(r)\right] r G_{2}\left(r, r^{\prime} ; E_{0}\right)=\frac{\delta\left(r^{\prime}-r\right)}{r} .}
\end{gathered}
$$

$V_{\text {cent }}(r)$ denotes the central part of the potential, $V_{\text {ten }}(r)$ the tensor potential. The indices of the Green's functions in Eqs. (B.12, B.13) reflect the orbital angular momentum state, whereas $J=1, S=1$ is not written down explicitly. Acting with the operator given in square brackets in Eq. (B.11) on $\chi_{\hat{C}}(r)$, the integral over $r^{\prime}$ collapses and we find

$$
\left[\frac{d^{2}}{d r^{2}}+m_{N}\left(E_{0}-V_{\hat{C}}(r)\right)-\frac{L_{C}\left(L_{C}+1\right)}{r^{2}}\right] r \chi_{\hat{C}}(r)=m_{N} u_{l^{\prime}}(r) J_{f}(r) .
$$

This is a second-order differential equation in $r$ with an inhomogeneity, which can be interpreted as a source term. Its solutions are real for $E_{0}<0$ and complex for $E_{0}>0$. The latter case corresponds to $\omega>B$, i.e. the photon carries enough energy to break up the deuteron into its two constituents. Obviously, an imaginary part only appears in the $s$-channel diagrams, where the incoming photon is absorbed before the other one is emitted. In Appendix $\mathrm{C}$ we will use the imaginary part of the amplitudes to derive the total deuteron-photodisintegration cross section via the optical theorem.

For $r \rightarrow \infty, u_{l^{\prime}}(r) J_{f}(r) \rightarrow 0$ due to $u_{l^{\prime}}(r) \rightarrow 0$, i.e. Eq. (B.14) reduces to a homogeneous differential equation. Furthermore, $V_{\hat{C}}(r) \rightarrow 0$ for $r \rightarrow \infty$. Therefore, we are for large distances left with

$$
\left[\frac{d^{2}}{d r^{2}}+E_{0} m_{N}-\frac{L_{C}\left(L_{C}+1\right)}{r^{2}}\right] r \chi_{\hat{C}}(r)=0 .
$$

This equation is known to be solved by a linear combination of the spherical Bessel functions of the first and second kind, $j_{L_{C}}(Q r)$ and $n_{L_{C}}(Q r)$ with $Q=\sqrt{m_{N} E_{0}}$. Note that $Q$ can be real or imaginary 2 , depending on $E_{0}$. In our case the boundary condition is that $\chi_{\hat{C}}(r)$ must be an outgoing spherical wave for large $r$, cf. e.g. [61]. Therefore we may write

$$
\lim _{r \rightarrow \infty} \chi_{\hat{C}}(r) \propto h_{L_{C}}^{(1)}(Q r)
$$

with $h_{L_{C}}^{(1)}(Q r)$ the spherical Hankel function of the first kind, defined as

$$
h_{L_{C}}^{(1)}(Q r)=j_{L_{C}}(Q r)+i n_{L_{C}}(Q r) .
$$

Once we have numerical solutions for the homogeneous and the inhomogeneous differential equation, we need to find the correct linear combination which satisfies the condition (B.16). In other words we have to determine the coefficient $\lambda$ which fulfills

$$
\lim _{r \rightarrow \infty}\left\{\chi_{\hat{C}}^{\mathrm{in}}(r)+\lambda \chi_{\hat{C}}^{\mathrm{hom}}(r)\right\} \propto h_{L_{C}}^{(1)}(Q r),
$$

\footnotetext{
${ }^{2}$ The sign of the imaginary solution is determined by adding an infinitesimal imaginary part to $-B$, i.e. $B \rightarrow B-i \epsilon$.
} 
where $\chi_{\hat{C}}^{\text {in }}(r)\left(\chi_{\hat{C}}^{\text {hom }}(r)\right)$ denote the solution to the inhomogeneous (homogeneous) differential equation. In the asymptotic limit, $\chi_{\hat{C}}(r)$ must be a linear combination of $j_{L_{C}}(Q r)$ and $n_{L_{C}}(Q r)$ or, equivalently, of $j_{L_{C}}(Q r)$ and $h_{L_{C}}^{(1)}(Q r)$. Therefore we can write the general solutions in the following way:

$$
\begin{aligned}
\chi_{\hat{C}}^{\text {hom }}(r) & =C_{\hat{C}}^{\text {hom }}(r)\left[j_{L_{C}}(Q r)+t_{\hat{C}}^{\text {hom }}(r) h_{L_{C}}^{(1)}(Q r)\right], \\
\chi_{\hat{C}}^{\mathrm{in}}(r) & =C_{\hat{C}}^{\mathrm{in}}(r)\left[j_{L_{C}}(Q r)+t_{\hat{C}}^{\mathrm{in}}(r) h_{L_{C}}^{(1)}(Q r)\right]
\end{aligned}
$$

with functions $C_{\hat{C}}^{\text {in/hom }}(r), t_{\hat{C}}^{\text {in/hom }}(r)$ which become the constants $C_{\hat{C}}^{\text {in/hom }}, t_{\hat{C}}^{\text {in/hom }}$ for large $r$. With the choice $\lambda=-C_{\hat{C}}^{\text {in }} / C_{\hat{C}}^{\text {hom }}$ we find

$$
\lim _{r \rightarrow \infty}\left\{\chi_{\hat{C}}^{\text {in }}(r)+\lambda \chi_{\hat{C}}^{\text {hom }}(r)\right\}=C_{\hat{C}}^{\text {in }}\left(t_{\hat{C}}^{\text {in }}-t_{\hat{C}}^{\text {hom }}\right) h_{L_{C}}^{(1)}(Q r),
$$

which satisfies the condition (B.16). Therefore we need to determine the coefficients $C_{\hat{C}}^{\text {in }}$, $C_{\hat{C}}^{\text {hom }}$. This has to be done in the region where $C_{\hat{C}}(r), t_{\hat{C}}(r)$ are constant, i.e. their derivatives vanish. In this region

$$
\ln \chi_{\hat{C}}^{\text {in } / \mathrm{hom}}(r)=\ln C_{\hat{C}}^{\text {in } / \text { hom }}+\ln \left[j_{L_{C}}(Q r)+t_{\hat{C}}^{\text {in } / \text { hom }} h_{L_{C}}^{(1)}(Q r)\right] .
$$

Defining $D^{\text {in } / \text { hom }}=\frac{d}{d r} \ln \chi_{\hat{C}}^{\text {in } / \text { hom }}=\frac{\chi_{\hat{C}}^{\text {in } / \text { hom }}}{\chi_{\hat{C}}^{\text {in hom }}}$ we find

$$
D^{\text {in } / \text { hom }}=\frac{d}{d r} \ln \left[j_{L_{C}}(Q r)+t_{\hat{C}}^{\text {in } / \text { hom }} h_{L_{C}}^{(1)}(Q r)\right]=\frac{\frac{d}{d r} j_{L_{C}}(Q r)+t_{\hat{C}}^{\text {in } / \text { hom }} \frac{d}{d r} h_{L_{C}}^{(1)}(Q r)}{j_{L_{C}}(Q r)+t_{\hat{C}}^{\text {in hom }} h_{L_{C}}^{(1)}(Q r)} .
$$

From this equation we can easily determine $t_{\hat{C}}^{\text {in/hom }}$, which we use to solve Eqs. (B.19, B.20) for $C_{\hat{C}}^{\text {in/hom }}$ and thus to determine $\lambda$.

Numerically, this is one of the most involved parts of this work. Fortunately, a nice and valuable cross-check to the routine can be performed. For this we consider again the double integral to be calculated, Eq. (B.6). This integral is obviously invariant under the interchange $r \leftrightarrow r^{\prime}$. A general feature of Green's functions is that they are symmetric under $r \leftrightarrow r^{\prime}$, i.e. $G_{\hat{C}}\left(r^{\prime}, r ; E_{0}\right)=G_{\hat{C}}\left(r, r^{\prime} ; E_{0}\right)$. Therefore,

$$
\mathcal{I}_{f i}^{l l^{\prime} \hat{C}}=\iint r^{\prime} d r^{\prime} r d r u_{l^{\prime}}(r) J_{f}(r) G_{\hat{C}}\left(r, r^{\prime} ; E_{0}\right) J_{i}\left(r^{\prime}\right) u_{l}\left(r^{\prime}\right) .
$$

This expression is identical to $\mathcal{I}_{f i}^{l l^{\prime}} \hat{C}$ with $i \leftrightarrow f, l \leftrightarrow l^{\prime}$, i.e. our results must be symmetric under $i \leftrightarrow f, l \leftrightarrow l^{\prime}$. This is a non-trivial check, because for $J_{f}(r) \neq J_{i}(r)$ completely different functions $\chi_{f}^{l^{\prime}} \hat{C}(r)$ are generated. Our routine agrees well with this symmetry - the deviation caused by numerical uncertainties is less than $1 \%$.

Now all tools to calculate $\mathcal{M}_{f i}^{\phi \phi 1,2}$ are prepared. However, as the algebraic manipulations are not too complicated, we refer the interested reader to Ref. [40] for further evaluation and the analytic results. There we also compute $\mathcal{M}_{f i}^{\phi \phi 3}$ and $\mathcal{M}_{f i}^{\phi \phi 4}$. 
We turn now to the evaluation of the subleading terms of Section 2.2.3, where we make use of the continuity equation (2.8) at only one or even none of the two vertices. Therefore, we have to specify the current $\vec{J}(\vec{\xi})$ and the relevant parts of the photon field $\vec{A}(\vec{\xi})$. These are the non-gradient terms in Eq. (2.6), i.e. $\vec{A}^{(1)}$ (Eq. (A.4)) and $\vec{A}^{(2)}$ (Eq. (A.5)). The onebody current is considered first. It consists of two parts, $\vec{J}^{(\sigma)}$ and $\vec{J}^{(p)}$, cf. Eqs. (2.25, 2.26). All possible combinations of $\vec{A}^{(1)}, \vec{A}^{(2)}$ and $\vec{J}^{(\sigma)}, \vec{J}^{(p)}$ have been calculated in 25, 26]. We also evaluated all these amplitudes, however we found that only $\vec{J}^{(\sigma)}$ gives visible contributions to the deuteron Compton cross sections. Therefore, we may restrict ourselves to the following combinations: $\left(\vec{J}^{(\sigma)}, \vec{A}^{(1)}\right)$, denoted by $\sigma 1$, and $\left(\vec{J}^{(\sigma)}, \vec{A}^{(2)}\right)$, denoted by $\sigma 2$.

We now calculate $\int \vec{J}^{(\sigma)}(\vec{\xi}) \cdot \vec{A}^{(1,2)}(\vec{\xi}) d^{3} \xi$. We start with the derivation for $\vec{A}^{(1)}$, writing only the $\xi$-dependent terms for simplicity.

$$
\begin{aligned}
\int \vec{J}^{(\sigma)}(\vec{\xi}) \cdot \vec{A}^{(1)}(\vec{\xi}) d^{3} \xi & \propto \int \sum_{j=n, p}\left[\vec{\nabla}_{\xi} \times \mu_{j} \vec{\sigma}_{j} \delta\left(\vec{\xi}-\vec{x}_{j}\right)\right] j_{L}(\omega \xi) \vec{L} Y_{L M}(\hat{\xi}) d^{3} \xi \\
& =\int \sum_{j=n, p} \mu_{j} \vec{\sigma}_{j} \delta\left(\vec{\xi}-\vec{x}_{j}\right) \vec{\nabla}_{\xi} \times\left(j_{L}(\omega \xi) \vec{L} Y_{L M}(\hat{\xi})\right) d^{3} \xi
\end{aligned}
$$

where one partial integration has been performed. Now we evaluate the integral and afterwards replace $\vec{x}_{p} \rightarrow \frac{\vec{r}}{2}, \vec{x}_{n} \rightarrow-\frac{\vec{r}}{2}$, cf. Eq. (2.14), yielding

$$
\int \vec{J}^{(\sigma)}(\vec{\xi}) \cdot \vec{A}^{(1)}(\vec{\xi}) d^{3} \xi \propto 2\left[\vec{\nabla}_{r} \times\left(j_{L}\left(\frac{\omega r}{2}\right) \vec{L} Y_{L M}(\hat{r})\right)\right]\left(\mu_{p} \vec{\sigma}_{p}-(-1)^{L} \mu_{n} \vec{\sigma}_{n}\right),
$$

where we used $Y_{L M}(-\hat{r})=(-1)^{L} Y_{L M}(\hat{r})$. By the help of the identity $\vec{T}_{L L M}(\hat{r})=\frac{1}{\sqrt{L(L+1)}}$. $\vec{L} Y_{L M}(\hat{r})$, see e.g. [62], this becomes

$$
\int \vec{J}^{(\sigma)}(\vec{\xi}) \cdot \vec{A}^{(1)}(\vec{\xi}) d^{3} \xi \propto 2 \sqrt{L(L+1)}\left[\vec{\nabla}_{r} \times j_{L}\left(\frac{\omega r}{2}\right) \vec{T}_{L L M}(\hat{r})\right]\left(\mu_{p} \vec{\sigma}_{p}-(-1)^{L} \mu_{n} \vec{\sigma}_{n}\right) .
$$

Now we can use the curl formula [62] for simplifying $\left[\vec{\nabla}_{r} \times j_{L}\left(\frac{\omega r}{2}\right) \vec{T}_{L L M}(\hat{r})\right]$ and the recursion relations for spherical Bessel functions to write

$$
\begin{aligned}
\int \vec{J}^{(\sigma)}(\vec{\xi}) \cdot \vec{A}^{(1)}(\vec{\xi}) d^{3} \xi & \propto\left[i \omega j_{L-1}\left(\frac{\omega r}{2}\right) \sqrt{\frac{L(L+1)^{2}}{2 L+1}} \vec{T}_{L L-1 M}(\hat{r})\right. \\
& \left.-i \omega j_{L+1}\left(\frac{\omega r}{2}\right) \sqrt{\frac{L^{2}(L+1)}{2 L+1}} \vec{T}_{L L+1 M}(\hat{r})\right]\left(\mu_{p} \vec{\sigma}_{p}-(-1)^{L} \mu_{n} \vec{\sigma}_{n}\right) .
\end{aligned}
$$

We found, like the authors of Ref. [25, 26], that the numerical importance of the various contributions rapidly decreases with increasing multipolarity $L$. Therefore, the term proportional to $\vec{T}_{L L+1 M}(\hat{r})$ may be neglected. Defining $\vec{S}=\frac{\vec{\sigma}_{p}+\vec{\sigma}_{n}}{2}$ and $\vec{t}=\frac{\vec{\sigma}_{p}-\vec{\sigma}_{n}}{2}$ and including 
all prefactors, we get the result

$$
\begin{aligned}
\int \vec{J}^{(\sigma)}(\vec{\xi}) \cdot \vec{A}^{(1)}(\vec{\xi}) d^{3} \xi= \\
\quad-\sum_{\vec{k}, \lambda= \pm 1} \sum_{L=1}^{\infty} \sum_{M=-L}^{L} \lambda \sqrt{2 \pi(L+1)} \frac{e \omega}{2 m_{N}} i^{L+1} j_{L-1}\left(\frac{\omega r}{2}\right) \vec{T}_{L L-1 M}(\hat{r}) \\
\quad \times\left[\left(\mu_{p}-(-1)^{L} \mu_{n}\right) \vec{S}+\left(\mu_{p}+(-1)^{L} \mu_{n}\right) \vec{t}\right] \cdot\left[a_{\vec{k}, \lambda} \delta_{M, \lambda}-a_{\vec{k}, \lambda}^{\dagger}(-1)^{L+\lambda} d_{M,-\lambda}^{L}(\theta)\right] .
\end{aligned}
$$

The scalar products are replaced using the relation

$$
\vec{T}_{J L M}(\hat{r}) \cdot \vec{V}=\left[Y_{L} \otimes V\right]_{J M},
$$

which holds for any vector (rank 1$)$ operator $(\otimes$ denotes the irreducible tensor product). An explicit proof of the relation (B.30) is given e.g. in Ref. [40]. We use it to finally rewrite Eq. (B.29) as

$$
\begin{aligned}
\int \vec{J}^{(\sigma)}(\vec{\xi}) \cdot \vec{A}^{(1)}(\vec{\xi}) d^{3} \xi & =-\sum_{\vec{k}, \lambda= \pm 1} \sum_{L=1}^{\infty} \sum_{M=-L}^{L} \lambda \sqrt{2 \pi(L+1)} \frac{e \omega}{2 m_{N}} i^{L+1} j_{L-1}\left(\frac{\omega r}{2}\right) \\
& \times\left\{\left(\mu_{p}-(-1)^{L} \mu_{n}\right)\left[Y_{L-1} \otimes S\right]_{L M}+\left(\mu_{p}+(-1)^{L} \mu_{n}\right)\left[Y_{L-1} \otimes t\right]_{L M}\right\} \\
& \times\left[a_{\vec{k}, \lambda} \delta_{M, \lambda}-a_{\vec{k}, \lambda}^{\dagger}(-1)^{L+\lambda} d_{M,-\lambda}^{L}(\theta)\right] .
\end{aligned}
$$

We turn now to the calculation of $\int \vec{J}^{(\sigma)}(\vec{\xi}) \cdot \vec{A}^{(2)}(\vec{\xi}) d^{3} \xi$. Again we restrict ourselves in the derivation to the $\xi$-dependent terms, finding

$$
\begin{aligned}
\int \vec{J}^{(\sigma)}(\vec{\xi}) \cdot \vec{A}^{(2)}(\vec{\xi}) d^{3} \xi & \propto \int \sum_{j=n, p}\left[\vec{\nabla}_{\xi} \times \mu_{j} \vec{\sigma}_{j} \delta\left(\vec{\xi}-\vec{x}_{j}\right)\right] \vec{\xi} j_{L}(\omega \xi) Y_{L M}(\hat{\xi}) d^{3} \xi \\
& =\vec{\nabla}_{r} \times\left(\vec{r} j_{L}\left(\frac{\omega r}{2}\right) Y_{L M}(\hat{r})\right)\left(\mu_{p} \vec{\sigma}_{p}+(-1)^{L} \mu_{n} \vec{\sigma}_{n}\right)
\end{aligned}
$$

where we have performed the same steps as in the derivation of Eq. (B.26). Using the relation

$$
\vec{r} j_{L}\left(\frac{\omega r}{2}\right) Y_{L M}(\hat{r})=\sqrt{L(L+1)} r j_{L}\left(\frac{\omega r}{2}\right)\left[\frac{\vec{T}_{L L-1 M}(\hat{r})}{\sqrt{(L+1)(2 L+1)}}-\frac{\vec{T}_{L L+1 M}(\hat{r})}{\sqrt{L(2 L+1)}}\right],
$$

which is derived e.g. in [40], and the curl formula we find

$$
\vec{\nabla}_{r} \times\left(r j_{L}\left(\frac{\omega r}{2}\right)\left[\frac{\vec{T}_{L L-1 M}(\hat{r})}{\sqrt{(L+1)(2 L+1)}}-\frac{\vec{T}_{L L+1 M}(\hat{r})}{\sqrt{L(2 L+1)}}\right]\right)=-i j_{L}\left(\frac{\omega r}{2}\right) \vec{T}_{L L M}(\hat{r}) .
$$

Combining Eqs. (B.32 B.34), together with the definitions of $\vec{S}$ and $\vec{t}$, cf. Eq. (B.29), yields

$$
\begin{aligned}
\int \vec{J}^{(\sigma)}(\vec{\xi}) \cdot \vec{A}^{(2)}(\vec{\xi}) d^{3} \xi & \propto-i \sqrt{L(L+1)} j_{L}\left(\frac{\omega r}{2}\right) \vec{T}_{L L M}(\hat{r}) \\
& \times\left[\left(\mu_{p}+(-1)^{L} \mu_{n}\right) \vec{S}+\left(\mu_{p}-(-1)^{L} \mu_{n}\right) \vec{t}\right] .
\end{aligned}
$$


Including all prefactors, we get

$$
\begin{aligned}
& \int \vec{J}^{(\sigma)}(\vec{\xi}) \cdot \vec{A}^{(2)}(\vec{\xi}) d^{3} \xi=-\sum_{\vec{k}, \lambda= \pm 1} \sum_{L=1}^{\infty} \sum_{M=-L}^{L} \sqrt{2 \pi(2 L+1)} \frac{e \omega}{2 m_{N}} i^{L} j_{L}\left(\frac{\omega r}{2}\right)\left\{\left(\mu_{p}+(-1)^{L} \mu_{n}\right)\right. \\
& \left.\times\left[Y_{L} \otimes S\right]_{L M}+\left(\mu_{p}-(-1)^{L} \mu_{n}\right)\left[Y_{L} \otimes t\right]_{L M}\right\} \cdot\left[a_{\vec{k}, \lambda} \delta_{M, \lambda}-a_{\vec{k}, \lambda}^{\dagger}(-1)^{L+\lambda} d_{M,-\lambda}^{L}(\theta)\right],
\end{aligned}
$$

making use of Eq. (B.30) once more.

We are now ready to calculate the amplitudes $\mathcal{M}_{f i}^{\phi \sigma 1}$ and $\mathcal{M}_{f i}^{\phi \sigma 2}$. The results are given in Ref. [40], together with the amplitudes $\mathcal{M}_{f i}^{\sigma 1 \sigma 1}$ and $\mathcal{M}_{f i}^{\sigma 2 \sigma 2}$, which do not contain the gradient part of the photon field. Nevertheless, these contributions are strong, cf. Fig. 11, due to the numerically large factor $\left(\mu_{p}-\mu_{n}\right)^{2} \approx 22$ which appears in those amplitudes. As the terms with both photons coupling to the current $\vec{J}^{(p)}$, Eq. (2.26), are not supported by this factor, these contributions are tiny and are not considered in our work. The mixed amplitudes $\mathcal{M}_{f i}^{\sigma 1 \sigma 2}, \mathcal{M}_{f i}^{\sigma 2 \sigma 1}$ also turn out negligibly small, cf. Ref. [40].

So far we (explicitly) only considered one-body currents. However, there are also nonnegligible contributions from pion-exchange currents, Fig. 7. The corresponding expressions in coordinate-space representation are (with $\vec{x}_{1}, \vec{x}_{2}$ the position of nucleon 1 and 2 , respectively)

$$
\vec{J}_{\mathrm{stat}}^{\mathrm{KR}}\left(\vec{\xi} ; \vec{x}_{1}, \vec{x}_{2}\right)=\frac{e f^{2}}{m_{\pi}^{2}}\left(\vec{\tau}_{1} \times \vec{\tau}_{2}\right)_{z}\left[\vec{\sigma}_{1} \delta\left(\vec{x}_{1}-\vec{\xi}\right)\left(\vec{\sigma}_{2} \cdot \hat{r}\right)+\vec{\sigma}_{2} \delta\left(\vec{x}_{2}-\vec{\xi}\right)\left(\vec{\sigma}_{1} \cdot \hat{r}\right)\right] \frac{\partial}{\partial r} \frac{\mathrm{e}^{-m_{\pi} r}}{r}
$$

for the Kroll-Ruderman (pair) current (Fig. 7(a)), and

$$
\vec{J}_{\text {stat }}^{\text {pole }}\left(\vec{\xi} ; \vec{x}_{1}, \vec{x}_{2}\right)=-\frac{e f^{2}}{4 \pi}\left(\vec{\tau}_{1} \times \vec{\tau}_{2}\right)_{z} \cdot\left(\vec{\nabla}_{1}-\vec{\nabla}_{2}\right)\left(\vec{\sigma}_{1} \cdot \vec{\nabla}_{1}\right)\left(\vec{\sigma}_{2} \cdot \vec{\nabla}_{2}\right) \frac{\mathrm{e}^{-m_{\pi}\left|\vec{x}_{1}-\vec{\xi}\right|}}{m_{\pi}\left|\vec{x}_{1}-\vec{\xi}\right|} \frac{\mathrm{e}^{-m_{\pi}\left|\vec{x}_{2}-\vec{\xi}\right|}}{m_{\pi}\left|\vec{x}_{2}-\vec{\xi}\right|}
$$

for the so-called pion-pole current (Fig. $7(\mathrm{~b})$ ), cf. e.g. [55] $]^{3}$. The relative vector $\vec{r}$ is defined as $\vec{r}=\vec{x}_{1}-\vec{x}_{2}$.

Our numerical evaluations show that the explicit inclusion of the pole current is well negligible in the process and energies under consideration. Therefore we are only concerned with the Kroll-Ruderman current, Eq. (B.37). This expression, however, is derived in the limit of static nucleons (denoted by the index 'stat'), i.e. the correction due to the photon energy is neglected. This being a rather crude approximation for $\omega \sim 100 \mathrm{MeV}$, which is close to the pion mass, we use

$$
\vec{J}^{\mathrm{KR}}\left(\vec{\xi} ; \vec{x}_{1}, \vec{x}_{2}\right)=\frac{e f^{2}}{m_{\pi}^{2}}\left(\vec{\tau}_{1} \times \vec{\tau}_{2}\right)_{z}\left[\vec{\sigma}_{1} \delta\left(\vec{x}_{1}-\vec{\xi}\right)\left(\vec{\sigma}_{2} \cdot \hat{r}\right)+\vec{\sigma}_{2} \delta\left(\vec{x}_{2}-\vec{\xi}\right)\left(\vec{\sigma}_{1} \cdot \hat{r}\right)\right] \frac{\partial}{\partial r} f^{\mathrm{KR}}(r)
$$

\footnotetext{
${ }^{3}$ Our convention for the pion-nucleon coupling $f^{2}$ differs by a factor $4 \pi$ from that used in [55].
} 
instead of Eq. (B.37) . The function $f^{\mathrm{KR}}(r)$ depends on the photon energy and is defined as

$$
f_{s}^{\mathrm{KR}}(r)=\frac{\mathrm{e}^{-m_{\pi} r}}{2 r}-2 \pi \int \frac{d^{3} q}{(2 \pi)^{3}} \frac{\mathrm{e}^{i \vec{q} \cdot\left(\vec{x}_{2}-\vec{x}_{1}\right)}}{\sqrt{m_{\pi}^{2}+\vec{q}^{2}}\left(\omega-\sqrt{m_{\pi}^{2}+\vec{q}^{2}}\right)}
$$

for an $s$-channel diagram and

$$
f_{u}^{\mathrm{KR}}(r)=\frac{\mathrm{e}^{-m_{\pi} r}}{2 r}-2 \pi \int \frac{d^{3} q}{(2 \pi)^{3}} \frac{\mathrm{e}^{i \vec{q} \cdot\left(\vec{x}_{2}-\vec{x}_{1}\right)}}{\sqrt{m_{\pi}^{2}+\vec{q}^{2}}\left(-\omega-\sqrt{m_{\pi}^{2}+\vec{q}^{2}}\right)}
$$

for the $u$-channel, cf. Ref. [40]. Note that for $\omega=0$, Eqs. (B.40) and (B.41) reduce to the above expression $f^{\mathrm{KR}}(r)=\frac{\mathrm{e}^{-m_{\pi} r}}{r}$.

Now we have another current at hand, which we can use to replace $\vec{J}(\vec{\xi})$ in Eq. (2.4). However, one has to be careful in order not to double-count certain contributions; e.g. it is not allowed to combine $H^{\text {int }}=-\int \vec{J}^{\mathrm{KR}}(\vec{\xi}) \cdot \vec{A}^{\text {full }}(\vec{\xi}) d^{3} \xi$ at one vertex with $H^{\text {int }}=$ $-\int \vec{J}(\vec{\xi}) \cdot \vec{\nabla} \phi(\vec{\xi}) d^{3} \xi$ at the other, as this contribution is already included - at least partly in the dominant terms, due to the use of Siegert's theorem, cf. discussion around Eq. (2.11). The Kroll-Ruderman current changes isospin, i.e. $H^{\text {int }}=-\int \vec{J}^{\mathrm{KR}}(\vec{\xi}) \cdot \vec{A}(\vec{\xi}) d^{3} \xi$ transforms the isospin-0 deuteron to an isospin-1 object. Therefore we need another isospin-changing interaction at the second vertex. Pauli's principle guarantees that the total wave function of the two-nucleon system has to be antisymmetric under the exchange of the two constituents. Stated differently, the wave function has to fulfill $(-1)^{S+L+T}=-1$, i.e. in order to have $T=$ 1 we need $S+L$ even. The operator that turned out to be the most important one numerically is $\left[Y_{0} \otimes t\right]_{1}$, cf. Eq. (B.31). The same observation has been made in [25, 26]. Nevertheless, also the operator $Y_{1}$, which stems from $\hat{\phi}_{i}, \hat{\phi}_{f}$, gives non-negligible contributions. However, in the amplitudes including $\hat{\phi}_{i}$ or $\hat{\phi}_{f}$ at the non-KR vertex, one is not allowed to use the full photon field in $H^{\text {int }}=-\int \vec{J}^{\mathrm{KR}}(\vec{\xi}) \cdot \vec{A}(\vec{\xi}) d^{3} \xi$, as explained after Eq. (B.39). Therefore we are left with $\mathcal{M}_{f i}^{\mathrm{KR} f u l l \sigma 1}, \mathcal{M}_{f i}^{\phi \mathrm{KR} 1}$ and $\mathcal{M}_{f i}^{\phi \mathrm{KR} 2}$, where 'KR full' denotes the integral over the Kroll-Ruderman current, multiplied by the full photon field. There is no danger of

double-counting $\mathcal{M}_{f i}^{\mathrm{KR} f u l l} \sigma 1$, as we only take into account the operator $\left[Y_{L-1} \otimes t\right]_{L}$. This operator, however, changes the deuteron spin, whereas the matrix elements arising from $\phi_{i, f}$ are spin-conserving. Further contributions, like the one where $\vec{J}(\vec{\xi})=\vec{J}^{\mathrm{KR}}(\vec{\xi})$ at both vertices, turned out to be small. The evaluation of the Kroll-Ruderman terms is given in Ref. [40], along with further details and the analytic results for all amplitudes.

\section{Total Deuteron-Photodisintegration Cross Section}

Besides complying with the low-energy theorem, cf. Section 2.2.4, another important check on our calculation is the extraction of the total deuteron-photodisintegration cross section from the Compton amplitude via the optical theorem. This process has been studied more extensively - experimentally as well as theoretically, see e.g. 64] - than elastic deuteron Compton scattering and there is plenty of data below $100 \mathrm{MeV}$ to compare with. 
The optical theorem in our normalization reads

$$
\sigma^{\mathrm{tot}}=\frac{1}{\omega} \cdot \frac{1}{6} \sum_{i=f} \operatorname{Im}\left[\mathcal{M}_{f i}(\theta=0)\right],
$$

i.e. the total cross section is the sum over the imaginary part of all deuteron Compton amplitudes in the forward direction with identical initial and final photon and deuteron states $\left(\lambda_{f}=\lambda_{i}, M_{f}=M_{i}\right)$, divided by the photon energy $\omega$. Like the elastic deuteron Compton cross section, this sum is divided by 6 , as we have to average over the initial states.

We calculate this cross section in the lab frame, in order to be able to compare to data. The rest of our work is performed in the $\gamma d$-cm frame, which has also been chosen in Ref. [40], and we refer to this reference for the final results of our amplitudes. Fortunately, these amplitudes are easily transformed into the lab frame. First we note that we only need to sum over the $s$-channel diagrams, as only they become complex for photon energies above the deuteron binding energy $B$, while the $u$-channel amplitudes stay real for all photon energies, cf. Section 2.2.2. As the authors of Ref. [25,26] calculate in the lab frame, we convert our calculation according to their work. In the $s$-channel the only change is $\omega+\frac{\omega^{2}}{2 m_{d}} \leftrightarrow \omega-\frac{\omega^{2}}{2 m_{d}}$, because in the lab frame, the deuteron's initial kinetic energy vanishes, whereas the total intermediate momentum is $\vec{P}_{C}=\vec{k}_{i}$. In the cm frame we have $\vec{P}_{i}=-\vec{k}_{i}$ and $\vec{P}_{C}=\overrightarrow{0}$ in the $s$-channel.

Our result for the total deuteron-photodisintegration cross section is shown in Fig. 19, together with data from [65] 72] which are described well by our calculation. In the lower left panel the low-energy regime is enlarged, in order to emphasize the non-vanishing value at threshold. The by far most important contribution at threshold stems from the singlet M1-transition of $\mathcal{M}_{f i}^{\sigma 1 \sigma 1}$, which is the amplitude with the magnetic part of the photon field $\vec{A}^{(1)}$, Eq. (A.4), coupling to the spin current at both vertices. It corresponds to the operator $\left[Y_{0} \otimes t\right]_{1}$, cf. Eq. (B.31), which transforms the deuteron into a (singlet) $S_{C}=0$-state. $M 1$ is the shorthand notation for the magnetic coupling of a photon with $L=1$. Nevertheless, as is well-known, already $1 \mathrm{MeV}$ above threshold the cross section is completely dominated by the amplitude $\mathcal{M}_{f i}^{\phi \phi 1}$ (Eq. (2.18) ), where for $L, L^{\prime}=1$ we have an E1-interaction at each vertex, and this dominance holds for all higher energies. In the lower right panel of Fig. 19, we show these two (most important) contributions to the total photodisintegration cross section, denoted as ' $E 1$ ' and ' $M 1$ '. We observe the well-known rise of ' $M 1$ ', as $\omega$ approaches the breakup threshold, cf. e.g. [73, 74], whereas ' $E 1$ ' is zero for $\omega=B$. Note that $E 1$ not only consists of $\mathcal{M}_{f i}^{\phi \phi 1}$ but of all amplitudes with an E1-interaction at the vertex of the incoming photon.

Strictly speaking there are also contributions from the one-body current $\vec{J}(p)(\vec{\xi})$, cf. Eq. (2.26). The corresponding amplitudes are given in [25, 26] but are not included in this work, as we found that their contributions to the elastic deuteron Compton cross sections are tiny (of the order of $1 \%$ ) and so is their effect on the total disintegration cross section. Nevertheless, in the high-energy regime of our calculation, say for $\omega \sim 100 \mathrm{MeV}$, they do give visible contributions to $E 1$, but these cancel nearly exactly against other terms which 

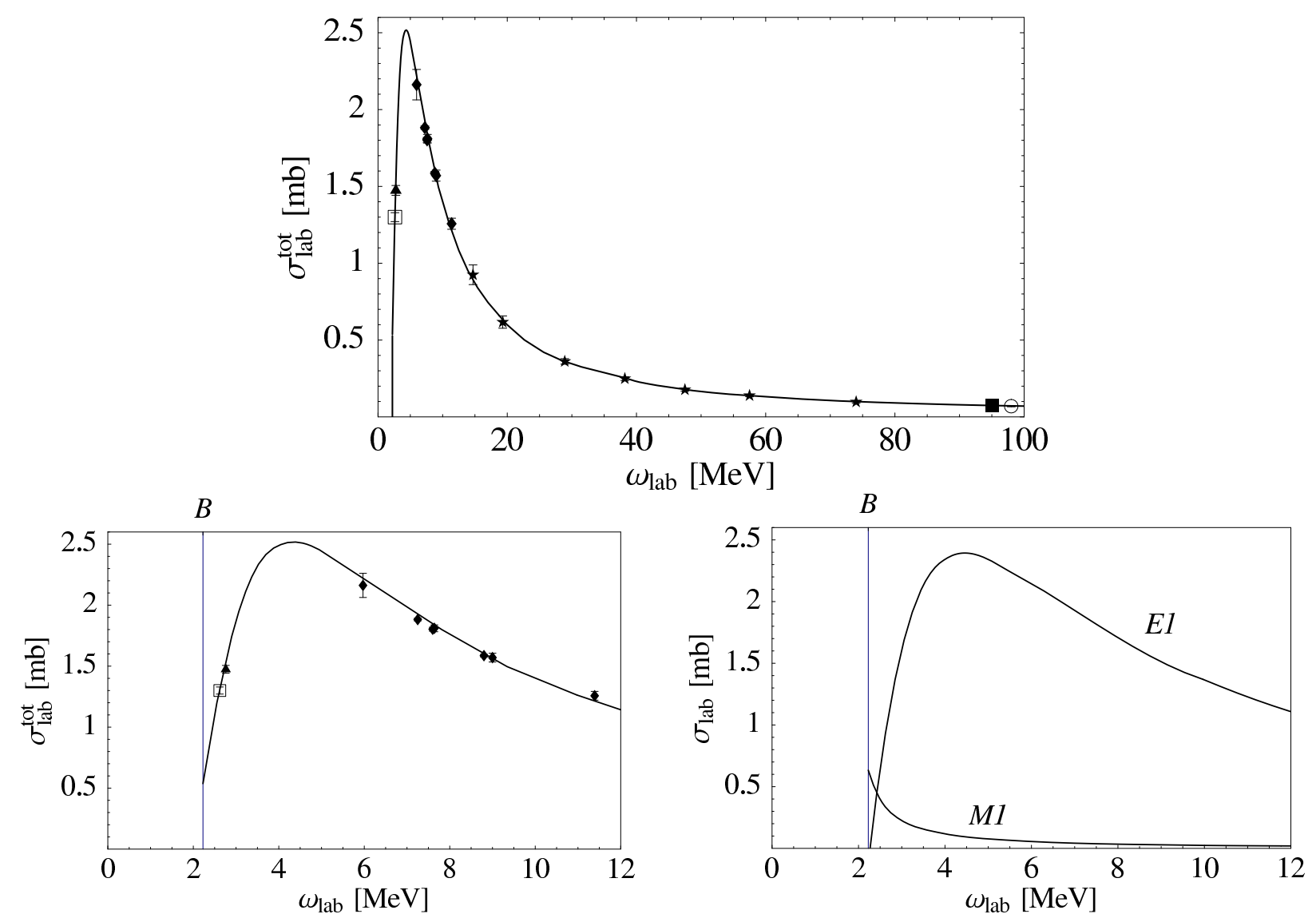

Figure 19: Total deuteron-photodisintegration cross section derived from our deuteron Compton amplitudes, together with data from [65] (open box), 66] (diamond), 67] (star), [68] (box), 69] (circle). The triangle corresponds to the weighted average of the data measured at $2.76 \mathrm{MeV}$ [70 72], as determined in [73]. ' $E 1$ ', ' $M 1$ ' denotes the contributions from the $E 1$ - and the singlet $M 1$-transition, respectively. $B$ is the binding energy of the deuteron.

also contain $\vec{J}^{(p)}$. Therefore, when we only look at the sum of all amplitudes contributing to $\sigma^{\text {tot }}$, we may well neglect the current $\vec{J}^{(p)}$.

We also compare our results with predictions for the strengths of electric and magnetic transitions close to threshold from the Effective Range Expansion [75, 76] given by

$$
\begin{gathered}
\sigma_{\mathrm{ER}}^{\mathrm{el}}=\frac{2}{3} \frac{e^{2}}{\gamma^{2}} \frac{\left(\frac{\omega}{B}-1\right)^{3 / 2}}{\left(\frac{\omega}{B}\right)^{3}\left(1-\gamma r_{t}\right)}, \\
\sigma_{\mathrm{ER}}^{\mathrm{mag}}=\frac{1}{6} \frac{e^{2}}{m_{N}^{2}}\left(\mu_{p}-\mu_{n}\right)^{2} \frac{k \gamma}{k^{2}+\gamma^{2}} \frac{\left(1-\gamma a_{s}+\frac{1}{4} a_{s}\left(r_{s}+r_{t}\right) \gamma^{2}-\frac{1}{4} a_{s}\left(r_{s}-r_{t}\right) k^{2}\right)^{2}}{\left(1+k^{2} a_{s}^{2}\right)\left(1-\gamma r_{t}\right)}
\end{gathered}
$$

with $\gamma=\sqrt{m_{N} B}$. The final-state relative momentum is $k=\left|\vec{p}_{p}-\vec{p}_{n}\right| / 2=\sqrt{m_{N}(\omega-B)}$, and for the singlet scattering length $a_{s}$ and the singlet (triplet) effective range $r_{s}\left(r_{t}\right)$ we 
use $a_{s}=-23.749 \mathrm{fm}, r_{s}=2.81 \mathrm{fm}$ and $r_{t}=1.76 \mathrm{fm}$ given in [34]. The explicit form of Eqs. (C.2. [C.3) is adopted from [73].

In Fig. 20, we compare our results with Eqs. (C.2, C.3), finding excellent agreement between both approaches. We also demonstrate - in the right panel - the non-negligible size of the KR diagrams. The only visible contributions to the total disintegration cross section at low energies including the Kroll-Ruderman current are the amplitudes $\mathcal{M}_{f i}^{\mathrm{KR} \sigma 1}$, which contribute to $\sigma^{\mathrm{mag}}$.
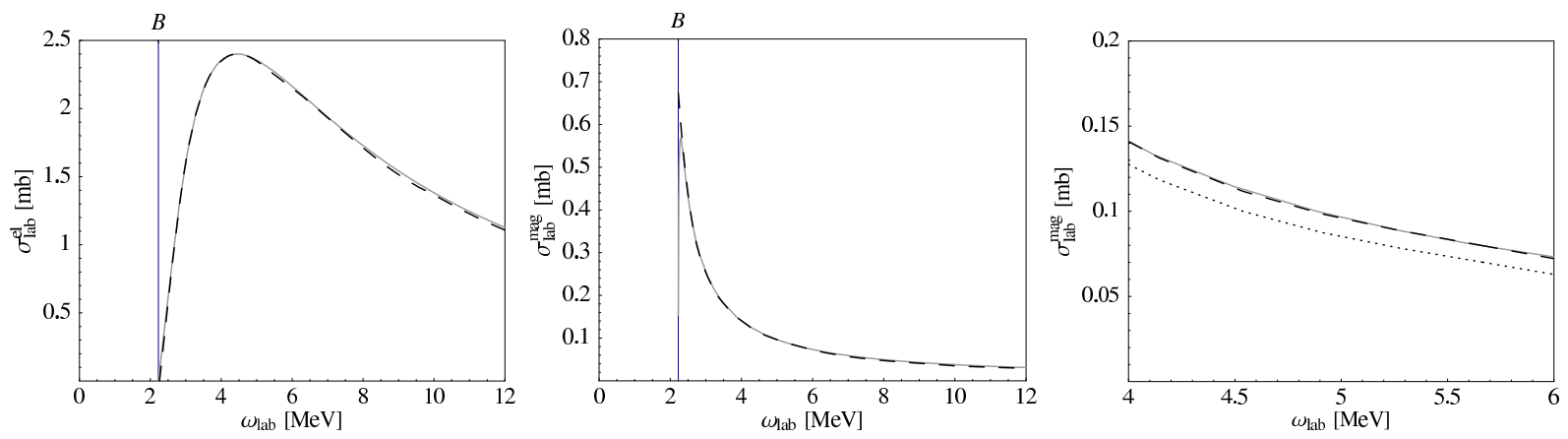

Figure 20: Comparison of our results (dashed) for the contributions of electric (left panel) and magnetic (middle and right panel) transitions to the total deuteron-photodisintegration cross section with predictions from the Effective Range Expansion (grey). The dotted curve in the right panel does not include the Kroll-Ruderman diagrams. 


\section{References}

[1] H.W. Grießhammer and T.R. Hemmert, Phys. Rev. C 65, 045207 (2002).

[2] R.P. Hildebrandt, H.W. Grießhammer, T.R. Hemmert and B. Pasquini, Eur. Phys. J. A 20, 293 (2004).

[3] M. Schumacher, Prog. Part. Nucl. Phys. 55, 567 (2005).

[4] Olmos de Leon et al., Eur. Phys. J. A 10, 207 (2001).

[5] T.R. Hemmert, B.R. Holstein and J. Kambor, Phys. Lett. B 395, 89 (1997).

[6] T.R. Hemmert, B.R. Holstein and J. Kambor, J. Phys. G 24, 1831 (1998).

[7] M. Lucas, Ph.D. thesis, University of Illinois (1994).

[8] M. Lundin et al., Phys. Rev. Lett. 90, 192501 (2003).

[9] D.L. Hornidge et al., Phys. Rev. Lett. 84, 2334 (2000).

[10] H. R. Weller, M. W. Ahmed, H. Gao, W. Tornow, Y. K. Wu, M. Gai and R. Miskimen, Prog. Part. Nucl. Phys. 62, 257 (2009).

[11] H. Weller et al., HIGS-E-18-09; H. Weller, private communication.

[12] R. Miskimen et al., HIGS-E-06-09; R. Miskimen, private communication.

[13] R. Miskimen, Measuring the Spin-Polarizabilities of the Proton at HI $\gamma$ S , presentation at the INT workshop on Soft Photons and Light Nuclei, 17 June 2008, and private communication.

[14] H. Gao et al., HIGS-E-07-10; H. Gao, private communication.

[15] M. Ahmed et al., HIGS-E-06-10; M. Ahmed, private communication.

[16] G. Feldman et al., Few Body Syst. 44, 325 (2008).

[17] MAXlab experiment NP-006 (http://www.maxlab.lu.se/kfoto/ExperimentalProgram/PAC_info.html G. Feldman, K. Fissum and L. Myers, private communication.

[18] A. Richter and P. von Neumann-Cosel, private communication (2005).

[19] R. Beck, Nucleon Compton Scattering at MAMI, talk at the INT workshop on Soft Photons and Light Nuclei, 17 June 2008; V. Ahrens and. R. M. Annand, private communication.

[20] M.I. Levchuk and A.I. L'vov, Nucl. Phys. A 674, 449 (2000).

[21] K. Kossert et al., Eur. Phys. J. A 16, 259 (2003). 
[22] R.P. Hildebrandt, H.W. Grießhammer, T.R. Hemmert and D.R. Phillips, Nucl. Phys. A 748, 573 (2005).

[23] V. Bernard, N. Kaiser, J. Kambor and U.-G. Meißner, Nucl. Phys. B 388, 315 (1992).

[24] D.R. Phillips, J. Phys. G 36, 104004 (2009).

[25] J.J. Karakowski and G.A. Miller, Phys. Rev. C 60, 014001 (1999).

[26] J.J. Karakowski, Ph.D. thesis, preprint nt@uw-99-6 (1999) [nucl-th/9901011] .

[27] S.R. Beane, M. Malheiro, D.R. Phillips and U. van Kolck, Nucl. Phys. A 656, 367 (1999).

[28] S.R. Beane, M. Malheiro, J.A. McGovern, D.R. Phillips and U. van Kolck, Phys. Lett. B 567, 200 (2003); erratum ibid. B 607, 320 (2005).

[29] S.R. Beane, M. Malheiro, J.A. McGovern, D.R. Phillips and U. van Kolck, Nucl. Phys. A 747,311 (2005).

[30] S. Weinberg, Phys. Lett. B 251, 288 (1990).

[31] S. Weinberg, Nucl. Phys. B 363, 3 (1991).

[32] V.G. Stoks, R.A. Klomp, C.P. Terheggen and J.J. de Swart, Phys. Rev. C 49, 2950 (1994).

[33] R. Machleidt, F. Sammarruca and Y. Song, Phys. Rev. C 53, 1483 (1996).

[34] R.B. Wiringa, V.G. Stoks and R. Schiavilla, Phys. Rev. C 51, 38 (1995).

[35] S.R. Beane, V. Bernard, E. Epelbaum, U.-G. Meißner and D.R. Phillips, Nucl. Phys. A 720,399 (2003).

[36] D.R. Phillips, Phys. Lett. B 567, 12 (2003).

[37] M. Rho, in Proceedings of the 10th Taiwan Nuclear Physics Spring School, Hualien, Taiwan, China (2002).

[38] A. Nogga, R.G.E. Timmermans and U. van Kolck, Phys. Rev. C 72, 054006 (2005).

[39] M. C. Birse, Phys. Rev. C 74, 014003 (2006) arXiv:nucl-th/0507077.

[40] R.P. Hildebrandt, Ph.D. thesis, TU München, mediaTUM_disshab_000000000003041 (2005) [nucl-th/0512064].

[41] H.W. Grießhammer, in preparation.

[42] H. W. Grießhammer, nucl-th/0611074]. 
[43] H. W. Grießhammer, arXiv:0710.2924 [nucl-th]].

[44] S.R. Beane and M.J. Savage, Nucl. Phys. A 694, 511 (2001).

[45] H.W. Grießhammer and G. Rupak, Phys. Lett. B 529, 57 (2002).

[46] J. Chen, X. Ji and Y. Li, Phys. Rev. C 71, 044321 (2005).

[47] J.L. Friar, Ann. of Phys. 95, 170 (1975).

[48] A.J.F. Siegert, Phys. Rev. 52, 787 (1937).

[49] H. Arenhövel, Z. Phys. A 297, 129 (1980).

[50] M. Weyrauch and H. Arenhövel, Nucl. Phys. A 408, 425 (1983).

[51] V. Bernard, N. Kaiser and U.-G. Meißner, Int. J. Mod. Phys. E 4, 193 (1995).

[52] T.R. Hemmert, B.R. Holstein, J. Kambor and G. Knöchlein: Phys. Rev. D 57, 5746 (1998).

[53] E. Epelbaum, W. Glöckle and U.-G. Meissner, Eur. Phys. J. A 19, 125 (2004); ibid. 19, 401 (2004).

[54] M.E. Rose, Elementary Theory of Angular Momentum, John Wiley \& Sons, inc., New York $\cdot$ London $\cdot$ Sydney (1957).

[55] T. Ericson and W. Weise, Pions and Nuclei, Clarendon Press, Oxford, ISBN 0-19852008-5 (1988).

[56] D.R. Phillips, J. Phys. G 34, 365 (2007).

[57] T.-S. Park, K. Kubodera, D.-P. Min and M. Rho, Nucl. Phys. A 646, 83 (1999).

[58] E. Epelbaum, W. Glöckle and U.-G. Meißner, Nucl. Phys. A 671, 295 (2000).

[59] J. A. McGovern, H. W. Grießhammer, D. R. Phillips and D. Shukla, arXiv:0910.1184 [nucl-th]].

[60] H. Grießhammer, J. McGovern, D. R. Phillips, D. Shukla, work in progress.

[61] J.J. Sakurai, Modern Quantum Mechanics, Addison-Wesley Publishing Company, ISBN 0-201-53929-2 (1994).

[62] A.R. Edmonds, Angular Momentum in Quantum Mechanics, University Press, Princeton, ISBN 0-691-07912-9 (1996).

[63] D.A. Varshalovich, A.N. Moskalev and V.K. Khersonskii, Quantum Theory of Angular Momentum, World Scientific, Singapore, ISBN 9971-50-107-4 (1988). 
[64] F. Partovi, Ann. Phys. 27, 79 (1964).

[65] P. Marin, G.R. Bishop and H. Halban, Proc. Phys. Soc. 67A, 1113 (1954).

[66] Y. Birenbaum et al., Phys. Rev. C 32, 1825 (1985).

[67] R. Bernabei et al., Phys. Rev. Lett. 57, 1542 (1986).

[68] H.O. Meyer et al., Phys. Rev. C 31, 309 (1985).

[69] E. de Sanctis et al., Phys. Rev. C 34, 413 (1986).

[70] R. Moreh, T.J. Kennett and W.V. Prestwick, Phys. Rev. C 39, 1247 (1989); erratum ibid. C 40, 1548 (1989).

[71] A.H. Snell, E.C. Barker and R.L. Sternberg, Phys. Rev. 80, 637 (1950).

[72] W.R. McMurray and C.H. Collie, Proc. Phys. Soc. 68A, 181 (1955).

[73] H. Arenhövel and M. Sanzone, Photodisintegration of the Deuteron, Few Body Systems Suppl. 3, Springer-Verlag Wien · New York, ISBN 3-211-82276-3 (1991).

[74] G.E. Brown and A.D. Jackson, The Nucleon-Nucleon Interaction, North-Holland Publishing Company, Amsterdam • Oxford, ISBN 0720403359 (1976).

[75] H.A. Bethe, Phys. Rev. 76, 38 (1949); J. Schwinger, hectographed notes on nuclear physics, Harvard University (1947).

[76] H.A. Bethe and C. Longmire, Phys. Rev. 77, 647 (1950). 